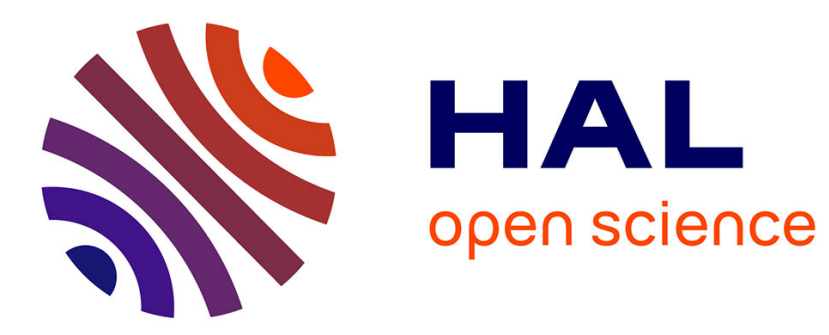

\title{
Calibration of a stock's beta using options prices
}

Sofiene El Aoud, Frédéric Abergel

\section{To cite this version:}

Sofiene El Aoud, Frédéric Abergel. Calibration of a stock's beta using options prices. Econophysics Kolkata conference, Mar 2014, Kolkata, India. hal-01006405

\section{HAL Id: hal-01006405 https://hal.science/hal-01006405}

Submitted on 16 Jun 2014

HAL is a multi-disciplinary open access archive for the deposit and dissemination of scientific research documents, whether they are published or not. The documents may come from teaching and research institutions in France or abroad, or from public or private research centers.
L'archive ouverte pluridisciplinaire HAL, est destinée au dépôt et à la diffusion de documents scientifiques de niveau recherche, publiés ou non, émanant des établissements d'enseignement et de recherche français ou étrangers, des laboratoires publics ou privés. 


\title{
Calibration of a stock's beta using options prices
}

\author{
Sofiene El Aoud ${ }^{\dagger *} \quad$ Frederic ABERGEL ${ }^{\dagger \dagger}$
}

February 28, 2014

\begin{abstract}
We present in our work a continuous time Capital Asset Pricing Model where the volatilities of the market index and the stock are both stochastic. Using a singular perturbation technique, we provide approximations for the prices of european options on both the stock and the index. These approximations are functions of the model parameters. We show then that existing estimators of the parameter beta, proposed in the recent literature, are biased in our setting because they are all based on the assumption that the idiosyncratic volatility of the stock is constant. We provide then an unbiased estimator of the parameter beta using only implied volatility data. This estimator is a forward measure of the parameter beta in the sense that it represents the information contained in derivatives prices concerning the forward realization of this parameter, we test then its capacity of prediction of forward beta and we draw a conclusion concerning its predictive power.
\end{abstract}

*sofiene.elaoud@ecp.fr

†Ecole Centrale Paris, Laboratoire de Mathématiques Appliquées aux Systèmes, Grande Voie des Vignes, 92290 Châtenay Malabry, France

${ }_{\ddagger}^{\ddagger}$ frederic.abergel@ecp.fr 


\section{Contents}

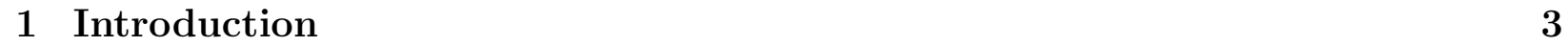

2 Summary of existing results in the literature $\quad 4$

2.1 The model of Fouque et $a l$. . . . . . . . . . . . . . . . . . . . 4

2.2 Calibration of implied beta . . . . . . . . . . . . . . . . . . . . . 5

2.3 Limits of the model . . . . . . . . . . . . . . . . . . . . . . . 5

\begin{tabular}{|lll}
3 & Model with fast mean-reverting idiosyncratic volatility & 6
\end{tabular}

3.1 Presentation of the model . . . . . . . . . . . . . . . . 6

3.2 Pricing options on the index and the stock . . . . . . . . . . . . . . 7

3.2.1 Approximation formula for index option price . . . . . . . . . . . . . 7

3.2.2 Approximation formula for stock option price . . . . . . . . . . . . . 8

3.3 Calibration of implied beta using options prices . . . . . . . . . . . . . . . . 14

3.3.1 Approximation formula of the implied volatility smile . . . . . . . . . . 14

3.3.2 Comparison with the model with constant idiosyncratic volatility . . 16

3.3 .3 Alternative method for the estimation . . . . . . . . . . . . . . 16

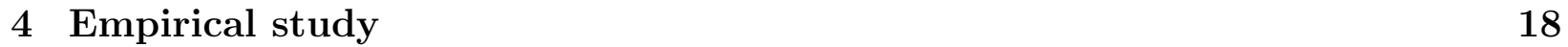

4.1 Numerical illustrations . . . . . . . . . . . . . . . . . . . . . . . . 18

4.2 Conclusion $\ldots \ldots \ldots \ldots$. . . . . . . . . . . . . . . . . . . . . . . 24

$\begin{array}{lll}5 & \text { Appendix } & 25\end{array}$

$5.1 \quad$ Appendix 1: Pricing options on the index . . . . . . . . . . . . . . . . 25

5.2 Appendix 2: Accuracy of the approximation . . . . . . . . . . . . . . . . 28 


\section{Introduction}

The notion of a stock's $\beta$ was first introduced in the theory of Capital Asset Pricing Model by Sharpe. This model extended previous works Markowitz did on portfolio construction theory (see [12] ). The CAPM model was considered to be original and innovative because it introduced the concept of systematic and specific risk and facilitated then the understanding of the equity market (see [5]). The parameter $\beta$, which is a key parameter in this model, enables to separate the stock risk into two parts. The first part represents the systematic risk implied by the market risk, while the second part is the idiosyncratic risk that reflects the specific performance of the stock. The parameter $\beta$ is of great use and its estimation is crucial in the construction of stock portfolios (see [16], [1], [13] ). This parameter was traditionally estimated using historical data of daily returns of the stock and the market index (see [18], [9]). In this approach, the estimator of the parameter is obtained as the slope of the linear regression of stock returns on market index returns. This approach is backwardlooking as it estimates the realized value of the parameter in the past using historical data. This characteristic can be considered as a weakness of the method. In fact, the value of the realized beta in the future can be remarkably different from its realization in the past, so the method lacks a predictive power.

In the recent literature, different authors have focused on the estimation of the $\beta$ coefficient using options data. This methodology provides a different way to estimate the parameter $\beta$. In fact, whereas classical methods allow an historical estimation of this parameter, the method based on the use of options prices enables us to obtain a "forward looking" measure of this parameter. In fact, the obtained estimator represents the information contained in derivatives prices and then summarizes the expectation of market participants for the forward realization of this parameter.

In [4], Christoffersen, Jacobs and Vainberg provided an estimation of this parameter using the risk-neutral variance and skewness of the stock and the index. More recently, Fouque and Kollman proposed in [6] a continuous-time CAPM model in which the market index has a stochastic volatility driven by a fast mean-reverting process. Using a singular perturbation method, they managed to obtain an approximation of the beta parameter depending on the skews of implied volatilities of both the stock and the index. Fouque and Tashman introduced also in [7] a "Stressed-Beta model" in which the parameter $\beta$ can take two values depending on the market regime. Using this model, Fouque et al provided a method to price options on index and stock. This method enables also to estimate the parameter $\beta$ based on options data. In [3], Carr and Madan used the CAPM model to price options on the stock when options on the index are liquid. Their approach didn't aim to estimate the parameter beta using option prices, but to price options on the stock given the parameter beta and options prices on the market index.

This work is inspired mainly from [6] and [10]. We look here into the estimation of the coefficient $\beta$ using options prices. The paper is organized as follows : in the first part, we make a brief reminder of the results obtained in the recent literature mainly in [6]. In the second part, we focus on the case where the index volatility and the stock's idiosyncratic 
volatility are both stochastic. This model is more likely to reproduce the stylised facts observed in the market and to capture realistic relations between the stock and the market index. We provide an estimator for the beta coefficient by means of a singular perturbation technique. In the third part, we make an empirical study in order to test the predictive power of our estimator for the forward realized $\beta$.

\section{Literature review}

\subsection{The model of Fouque et al}

Fouque et al proposed in [6] a continuous time Capital Asset Pricing Model in a stochastic volatility environment. They supposed that the volatility of the index is driven by a fast mean-reverting Ornstein-Uhlenbeck process. Under the historic probability measure $P$, we have :

$$
\begin{aligned}
\frac{d I_{t}}{I_{t}} & =\mu_{I} d t+f\left(Y_{t}\right) d W_{t}^{(1)} \\
\frac{d S_{t}}{S_{t}} & =\mu_{S} d t+\beta \frac{d I_{t}}{I_{t}}+\sigma d W_{t}^{(2)} \\
d Y_{t} & =\frac{1}{\epsilon}\left(m-Y_{t}\right) d t+\frac{\nu \sqrt{2}}{\sqrt{\epsilon}} d W_{t}^{(3)}
\end{aligned}
$$

where $W_{t}^{(3)}=\rho W_{t}^{(1)}+\sqrt{1-\rho^{2}} W_{t}^{(4)}$ and $W=\left(\begin{array}{l}W^{(1)} \\ W^{(2)} \\ W^{(4)}\end{array}\right)$ is a Wiener process under $P$

Let $\lambda_{t}=\left(\begin{array}{c}\frac{\mu_{I}-r}{f\left(Y_{t}\right)} \\ \frac{\mu_{S}+r(\beta-1)}{\sigma} \\ \gamma\left(Y_{t}\right)\end{array}\right), P^{*}$ be a probability measure equivalent to $P$ defined as:

$$
{\frac{d P^{*}}{d P_{\mid \mathcal{F}_{t}}}}=\exp \left(-\int_{0}^{t} \lambda_{u}^{\prime} d W_{u}-\frac{1}{2} \int_{0}^{t}\left|\lambda_{u}\right|^{2} d u\right)
$$

and $W^{*}=\left(\begin{array}{l}W^{*,(1)} \\ W^{*,(2)} \\ W^{*,(4)}\end{array}\right)$ such that: $W_{t}^{*}=W_{t}+\int_{0}^{t} \lambda_{u} d u$. By Girsanov's theorem, $W^{*}$ is a brownian motion under $P^{*}$.

Under continuity and boundedness conditions on the function $\gamma, P^{*}$ is a risk-neutral probabilitymeasure under which we have:

$$
\begin{aligned}
\frac{d I_{t}}{I_{t}} & =r d t+f\left(Y_{t}\right) d W_{t}^{*,(1)} \\
\frac{d S_{t}}{S_{t}} & =r d t+\beta f\left(Y_{t}\right) d W_{t}^{*,(1)}+\sigma d W_{t}^{*,(2)} \\
d Y_{t} & =\left(\frac{1}{\epsilon}\left(m-Y_{t}\right)-\frac{\nu \sqrt{2}}{\sqrt{\epsilon}} \chi\left(Y_{t}\right)\right) d t+\frac{\nu \sqrt{2}}{\sqrt{\epsilon}} d W_{t}^{*,(3)}
\end{aligned}
$$


where $\chi\left(Y_{t}\right)=\rho \frac{\mu_{I}-r}{f\left(Y_{t}\right)}+\sqrt{1-\rho^{2}} \gamma\left(Y_{t}\right)$ and $W_{t}^{*,(3)}=\rho W_{t}^{*,(1)}+\sqrt{1-\rho^{2}} W_{t}^{*,(4)}$.

We can notice that there is an infinity of risk-neutral probability measures. The choice of the function $\gamma$ determines the risk-neutral probability under which we price options.

\subsection{Calibration of implied beta}

Using a singular perturbation method with respect to the small parameter $\epsilon$, Fouque et al managed to obtain an approximation $\tilde{P}^{I, \epsilon}\left(K_{I}, T\right)$ for the price of an european call on the index with strike $K_{I}$ and maturity $T$, and an approximation $\tilde{P}^{S, \epsilon}\left(K_{S}, T\right)$ for the price of an european call on the stock with strike $K_{S}$ and maturity $T$. Afterwards, by doing a Taylor expansion in $\sqrt{\epsilon}$ for the implied volatility of the stock and the index, they managed to approximate the shape of the implied volatility surfaces as follows:

$$
\begin{aligned}
\Sigma_{I}\left(K_{I}, T\right) & =b_{I}+a_{I} \frac{\ln \left(\frac{F_{I}}{K_{I}}\right)}{T} \\
\Sigma_{S}\left(K_{S}, T\right) & =b_{S}+a_{S} \frac{\ln \left(\frac{F_{S}}{K_{S}}\right)}{T}
\end{aligned}
$$

where $F_{I}$ and $F_{S}$ are the forward prices for maturity $T$ of the index and stock respectively. The quantities $b_{I}, a_{I}, b_{S}, a_{S}$ are functions of the model parameters. The parameter $\beta$ can be approximated by $\hat{\beta}$ which is defined as :

$$
\hat{\beta}=\left(\frac{a_{S}}{a_{I}}\right)^{\frac{1}{3}} \frac{b_{S}}{b_{I}}
$$

\section{$2.3 \quad$ Limits of the model}

In the model described so far, Fouque et al made the assumption that the idiosyncratic volatility of the stock is constant. This hypothesis is too simplistic. In fact, a stock has an idiosyncratic volatility that varies significantly, especially when the market reacts to specific news of the company (earning expectation, restructuring projects,...). The idiosyncratic volatility has then its own dynamics (see [17], [2], [11], [15] ).

By way of example, we give here the graph of the idiosyncratic volatility $\sigma$ of XLF when projected on the SPX index, from 01/01/2008 to 31/12/2012. The parameter $\sigma$ is obtained here by computing the standard deviation of errors in the linear regression of stock's returns (XLF) on index returns (SPX) with a sliding window of 1 month. 


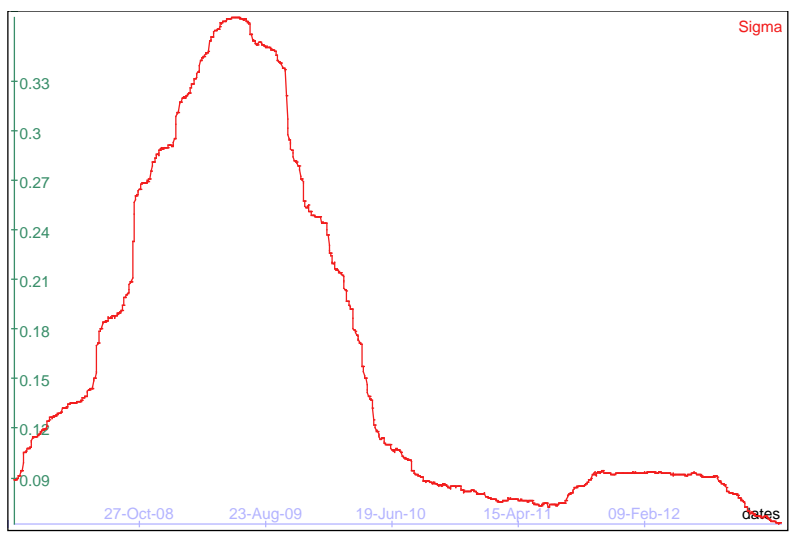

Figure 1: Evolution of the idiosyncratic volatility of XLF with respect to the SPX index The inspection of the graph above shows that the parameter $\sigma$ is not constant and then we should take this characteristic into consideration.

\section{Model with fast mean-reverting idiosyncratic volatil- ity}

In this section, we propose a new model in which the idyosyncratic volatility of the stock is driven by a fast mean-reverting Ornstein-Uhlenbeck process. We explain then how we could calibrate the parameter $\beta$ using options prices.

\subsection{Presentation of the model}

Let us assume that under the historic probability measure $P$, the stock and the index have the the following dynamics:

$$
\begin{aligned}
\frac{d I_{t}}{I_{t}} & =\mu_{I} d t+f_{1}\left(Y_{t}\right) d W_{t}^{(1)} \\
\frac{d S_{t}}{S_{t}} & =\mu_{S} d t+\beta \frac{d I_{t}}{I_{t}}+f_{2}\left(Z_{t}\right) d W_{t}^{(2)} \\
d Y_{t} & =\frac{1}{\epsilon}\left(m_{Y}-Y_{t}\right) d t+\frac{\nu_{Y} \sqrt{2}}{\sqrt{\epsilon}} d W_{t}^{(3)}, \\
d Z_{t} & =\frac{\alpha}{\epsilon}\left(m_{Z}-Z_{t}\right) d t+\frac{\nu_{Z} \sqrt{2 \alpha}}{\sqrt{\epsilon}} d W_{t}^{(4)}
\end{aligned}
$$

where $W_{t}^{(3)}=\rho_{Y} W_{t}^{(1)}+\sqrt{1-\rho_{Y}^{2}} W_{t}^{(5)}, W_{t}^{(4)}=\rho_{Z} W_{t}^{(2)}+\sqrt{1-\rho_{Z}^{2}} W_{t}^{(6)}$ and $W=\left(\begin{array}{l}W^{(1)} \\ W^{(2)} \\ W^{(5)} \\ W^{(6)}\end{array}\right)$ is a Wiener process under $P$. 
Let $\lambda_{t}=\left(\begin{array}{c}\frac{\mu_{I}-r}{f_{1}\left(Y_{t}\right)} \\ \underline{\mu_{S}+r(\beta-1)} \\ f_{2}\left(Z_{t}\right) \\ \gamma_{1}\left(Y_{t}\right) \\ \gamma_{2}\left(Z_{t}\right)\end{array}\right)$ and $P^{*}$ a probability measure equivalent to $P$ and defined such that:

$$
\frac{d P^{*}}{d P}=\exp \left(-\int_{0}^{t} \lambda_{u}^{\prime} d W_{u}-\frac{1}{2} \int_{0}^{t}\left|\lambda_{u}\right|^{2} d u\right) .
$$

Let us define $W^{*}=\left(\begin{array}{l}W^{*,(1)} \\ W^{*,(2)} \\ W^{*,(5)} \\ W^{*,(6)}\end{array}\right)$ such that $W_{t}^{*}=W_{t}+\int_{0}^{t} \lambda_{u} d u$. Using Girsanov's theorem, $W^{*}$ is a brownian motion under $P^{*}$.

$P^{*}$ is a risk-neutral probability measure under which we have:

$$
\begin{aligned}
\frac{d I_{t}}{I_{t}} & =r d t+f_{1}\left(Y_{t}\right) d W_{t}^{*,(1)} \\
\frac{d S_{t}}{S_{t}} & =r d t+\beta f_{1}\left(Y_{t}\right) d W_{t}^{*,(1)}+f_{2}\left(Z_{t}\right) d W_{t}^{*,(2)} \\
d Y_{t} & =\frac{1}{\epsilon}\left(m_{Y}-Y_{t}\right) d t-\frac{\nu_{Y} \sqrt{2}}{\sqrt{\epsilon}} \chi_{1}\left(Y_{t}\right) d t+\frac{\nu_{Y} \sqrt{2}}{\sqrt{\epsilon}} d W_{t}^{*,(3)}, \\
d Z_{t} & =\frac{\alpha}{\epsilon}\left(m_{Z}-Z_{t}\right) d t-\frac{\nu_{Z} \sqrt{2 \alpha}}{\sqrt{\epsilon}} \chi_{2}\left(Z_{t}\right) d t+\frac{\nu_{Z} \sqrt{2 \alpha}}{\sqrt{\epsilon}} d W_{t}^{*,(4)},
\end{aligned}
$$

where: $\chi_{1}\left(Y_{t}\right)=\rho_{Y} \frac{\mu_{I}-r}{f_{1}\left(Y_{t}\right)}+\sqrt{1-\rho_{Y}^{2}} \gamma_{1}\left(Y_{t}\right)$ and $\chi_{2}\left(Z_{t}\right)=\rho_{Z} \frac{\mu_{S}+r(\beta-1)}{f_{2}\left(Z_{t}\right)}+\sqrt{1-\rho_{Z}^{2}} \gamma_{2}\left(Z_{t}\right)$.

$W^{*,(3)}$ and $W^{*,(4)}$ are brownian motions under $P^{*}$ such that:

$$
\begin{aligned}
& W^{*,(3)}=\rho_{Y} W^{*,(1)}+\sqrt{1-\rho_{Y}^{2}} W^{*,(5)}, \\
& W^{*,(4)}=\rho_{Z} W^{*,(2)}+\sqrt{1-\rho_{Z}^{2}} W^{*,(6)} .
\end{aligned}
$$

\subsection{Pricing options on the index and the stock}

\subsubsection{Approximation formula for index option price}

We call $P^{I, \epsilon}\left(K_{I}, T\right)=E^{P^{*}}\left(e^{-r(T-t)}\left(I_{T}-K_{I}\right)^{+} \mid \mathcal{F}_{t}\right)$ the price of an european call on the index with strike $K_{I}$ and maturity $T$. We can easily see that the pricing of options on the index remains the same as in Fouque's model where the idiosyncratic volatility of the stock is constant. This is due to the fact that the diffusion equations of the processes $(I)$ and $(Y)$ are still the same.

By doing a singular perturbation method as in [6], Fouque et al obtained an approximation $\tilde{P}^{I, \epsilon}\left(K_{I}, T\right)$ for the price $P^{I, \epsilon}\left(K_{I}, T\right)$. For simplification purposes, we will use the notation 
$\tilde{P}^{I, \epsilon}$ instead of $\tilde{P}^{I, \epsilon}\left(K_{I}, T\right)$. The proof of the approximation result is given in $[6]$ and also in Appendix 1 for completeness. We just recall the result here.

$$
\tilde{P}^{I, \epsilon}=\tilde{P}_{0}^{I, \epsilon}-(T-t) V_{3}^{I, \epsilon} I_{t} \frac{\partial}{\partial I_{t}}\left(I_{t}^{2} \frac{\partial^{2} \tilde{P}_{0}^{I, \epsilon}}{\partial I_{t}^{2}}\right),
$$

where the quantities $\tilde{P}_{0}^{I, \epsilon}$ and $V_{3}^{I, \epsilon}$ are defined as:

$$
\begin{aligned}
\tilde{P}_{0}^{I, \epsilon} & =P_{B S}^{I}\left(\bar{\sigma}_{I}^{*}\right) \\
V_{2}^{I, \epsilon} & =-\frac{\sqrt{\epsilon}}{\sqrt{2}} \nu_{Y}<\phi_{I}^{\prime} \chi_{1}>_{1} \\
V_{3}^{I, \epsilon} & =\frac{\sqrt{\epsilon}}{\sqrt{2}} \rho_{Y} \nu_{Y}<\phi_{I}^{\prime} f_{1}>_{1} \\
\left(\bar{\sigma}_{I}^{*}\right)^{2} & =<f_{1}^{2}>_{1}-2 V_{2}^{I, \epsilon}
\end{aligned}
$$

We should precise here that $<.>_{1}$ is the average with respect to the invariant distribution of the Ornstein-Uhlenbeck process $\left(Y_{1}\right)$ whose dynamics are described by :

$$
d Y_{1, t}=\left(m_{Y}-Y_{1, t}\right) d t+\nu_{Y} \sqrt{2} d W_{t}^{(3)} .
$$

$\phi_{I}$ is defined as the solution of the following Poisson equation :

$$
\mathcal{L}_{0}^{I} \phi_{I}(y)=f_{1}^{2}(y)-<f_{1}^{2}>_{1}
$$

where $\mathcal{L}_{0}^{I}$ is the infinitesimal generator of the process $\left(Y_{1}\right)$ :

$$
\mathcal{L}_{0}^{I}=\frac{\partial}{\partial y}\left(m_{Y}-y\right)+\nu_{Y}^{2} \frac{\partial^{2}}{\partial y^{2}}
$$

\subsubsection{Approximation formula for stock option price}

Let $P_{t}^{S, \epsilon}\left(K_{S}, T\right)$ be the price at time $t$ of an european call on the stock with strike $K_{S}$ and maturity $T$ :

$$
P_{t}^{S, \epsilon}\left(K_{S}, T\right)=E^{P^{*}}\left(e^{-r(T-t)}\left(S_{T}-K_{S}\right)^{+} \mid \mathcal{F}_{t}\right)
$$

To simplify the notations, we will use the notation $P_{t}^{S, \epsilon}$ instead of $P_{t}^{S, \epsilon}\left(K_{S}, T\right)$.

Using a singular perturbation technique on the small parameter $\epsilon$, we can obtain an approximation $\tilde{P}^{S, \epsilon}$ for the option's price $P_{t}^{S, \epsilon}$. This approximation can be detailed as follows:

\section{Proposition 3.1}

$$
\tilde{P}^{S, \epsilon}=\tilde{P}_{0}^{S, \epsilon}-(T-t) V_{3}^{S, \epsilon} S_{t} \frac{\partial}{\partial S_{t}}\left(S_{t}^{2} \frac{\partial^{2} \tilde{P}_{0}^{S, \epsilon}}{\partial S_{t}^{2}}\right)
$$


where the quantities $\tilde{P}_{0}^{S, \epsilon}$ and $V_{3}^{S, \epsilon}$ are defined as:

$$
\begin{aligned}
\tilde{P}_{0}^{S, \epsilon} & =P_{B S}^{S}\left(t, S_{t}, \bar{\sigma}_{S}^{*}\right), \\
\left(\bar{\sigma}_{S}^{*}\right)^{2} & =\bar{\sigma}_{S}^{2}-2 V_{2}^{S, \epsilon}, \\
V_{2}^{S, \epsilon} & =-\frac{\sqrt{\epsilon}}{\sqrt{2}}\left(\beta^{2} \nu_{Y}<\phi_{I}^{\prime} \chi_{1}>+\nu_{Z} \sqrt{\alpha}<\phi_{\text {Idios }}^{\prime} \chi_{2}>\right), \\
V_{3}^{S, \epsilon} & =\frac{\sqrt{\epsilon}}{\sqrt{2}}\left(\beta^{3} \rho_{Y} \nu_{Y}<\phi_{I}^{\prime} f_{1}>+\rho_{Z} \nu_{Z} \sqrt{\alpha}<\phi_{\text {Idios }}^{\prime} f_{2}>\right) .
\end{aligned}
$$

Proof:

$$
P_{t}^{S, \epsilon}=E^{P^{*}}\left(e^{-r(T-t)}\left(S_{T}-K_{S}\right)^{+} \mid S_{t}=x, Y_{t}=y, Z_{t}=z\right)
$$

Using Itô's Lemma, we have:

$$
\mathcal{L}^{S} P_{t}^{S, \epsilon}=0
$$

We then expand $\mathcal{L}^{S}$ in powers of $\sqrt{\epsilon}$, and obtain:

$$
\mathcal{L}^{S}=\mathcal{L}_{2}^{S}+\frac{1}{\sqrt{\epsilon}} \mathcal{L}_{1}^{S}+\frac{1}{\epsilon} \mathcal{L}_{0}^{S},
$$

where:

$$
\begin{aligned}
\mathcal{L}_{0}^{S} & =\left(m_{Y}-y\right) \frac{\partial}{\partial y}+\nu_{Y}^{2} \frac{\partial^{2}}{\partial y^{2}}+\alpha\left(m_{Z}-z\right) \frac{\partial}{\partial z}+\alpha \nu_{Z}^{2} \frac{\partial^{2}}{\partial z^{2}} \\
\mathcal{L}_{1}^{S} & =-\nu_{Y} \sqrt{2} \chi_{1}(y) \frac{\partial}{\partial y}+\beta S_{t} f_{1}(y) \sqrt{2} \rho_{Y} \nu_{Y} \frac{\partial^{2}}{\partial S \partial y}-\nu_{Z} \sqrt{2 \alpha} \chi_{2}(z) \frac{\partial}{\partial z}+S_{t} f_{2}(z) \sqrt{2 \alpha} \rho_{Z} \nu_{Z} \frac{\partial^{2}}{\partial S \partial z} \\
\mathcal{L}_{2}^{S} & =\frac{\partial}{\partial t}+r\left(\frac{\partial}{\partial S} S_{t}-.\right)+\frac{1}{2} \frac{\partial^{2}}{\partial S_{t}^{2}} S_{t}^{2}\left(\beta^{2} f_{1}(y)^{2}+f_{2}(z)^{2}\right)
\end{aligned}
$$

We can note that $\mathcal{L}_{0}^{S}$ is the infinitesimal generator of the two-dimensional Ornstein-Uhlenbeck process $\left(\begin{array}{l}Y_{1} \\ Y_{2}\end{array}\right)$ having the following dynamics :

$$
d\left(\begin{array}{l}
Y_{1, t} \\
Y_{2, t}
\end{array}\right)=\left(\begin{array}{cc}
1 & 0 \\
0 & \alpha
\end{array}\right)\left(\left(\begin{array}{l}
m_{Y} \\
m_{Z}
\end{array}\right)-\left(\begin{array}{l}
Y_{1, t} \\
Y_{2, t}
\end{array}\right)\right) d t+\sqrt{2}\left(\begin{array}{cc}
\nu_{Y} & 0 \\
0 & \sqrt{\alpha} \nu_{Z}
\end{array}\right) d\left(\begin{array}{l}
W_{t}^{(3)} \\
W_{t}^{(4)}
\end{array}\right) .
$$

We define here:

- $\left\langle.>_{1}\right.$ denotes the averaging with respect to the invariant distribution of the process $\left(Y_{1, t}\right)_{t}$.

- $\left\langle.>_{2}\right.$ denotes the averaging with respect to the invariant distribution of the process $\left(Y_{2, t}\right)_{t}$.

- $<.>_{1,2}$ denotes the averaging with respect to the invariant distribution of $\left(\begin{array}{l}Y_{1, t} \\ Y_{2, t}\end{array}\right)_{t}$. 
We formally expand $P^{S, \epsilon}$ in powers of $\sqrt{\epsilon}$ :

$$
P^{S, \epsilon}=\sum_{i=0}^{\infty}(\sqrt{\epsilon})^{i} P_{i}^{S, \epsilon}
$$

then, we expand the term $\mathcal{L}^{S} P_{t}^{S, \epsilon}$ :

$$
\left(\mathcal{L}_{2}^{S}+\frac{1}{\sqrt{\epsilon}} \mathcal{L}_{1}^{S}+\frac{1}{\epsilon} \mathcal{L}_{0}^{S}\right)\left(\sum_{i=0}^{\infty}(\sqrt{\epsilon})^{i} P_{i}^{S, \epsilon}\right)=0
$$

By classifying the terms of the last equation by powers of $\sqrt{\epsilon}$, we obtain :

$$
\begin{aligned}
& \text { (0) : } \mathcal{L}_{2}^{S} P_{0}^{S, \epsilon}+\mathcal{L}_{1}^{S} P_{1}^{S, \epsilon}+\mathcal{L}_{0}^{S} P_{2}^{S, \epsilon}=0, \\
& (-1): \mathcal{L}_{1}^{S} P_{0}^{S, \epsilon}+\mathcal{L}_{0}^{S} P_{1}^{S, \epsilon}=0, \\
& (-2): \mathcal{L}_{0}^{S} P_{0}^{S, \epsilon}=0 \text {, } \\
& \text { (1) : } \mathcal{L}_{2}^{S} P_{1}^{S, \epsilon}+\mathcal{L}_{1}^{S} P_{2}^{S, \epsilon}+\mathcal{L}_{0}^{S} P_{3}^{S, \epsilon}=0, \\
& \text { (2) : } \mathcal{L}_{2}^{S} P_{2}^{S, \epsilon}+\mathcal{L}_{1}^{S} P_{3}^{S, \epsilon}+\mathcal{L}_{0}^{S} P_{4}^{S, \epsilon}=0 \text {. }
\end{aligned}
$$

The term of order $(-2)$ in $\sqrt{\epsilon}$ states that $\mathcal{L}_{0}^{S} P_{0}^{S, \epsilon}=0$. Given that $\mathcal{L}_{0}^{S}$ contains only derivatives with respect to $y$ and $z$, we can solve this equation by choosing $P_{0}^{S, \epsilon}=P_{0}^{S, \epsilon}\left(t, S_{t}\right)$ independent of $Y_{t}$ and $Z_{t}$.

The term of order $(-1)$ in $\sqrt{\epsilon}$ states that $\mathcal{L}_{1}^{S} P_{0}^{S, \epsilon}+\mathcal{L}_{0}^{S} P_{1}^{S, \epsilon}=0 . \mathcal{L}_{1}^{S}$ contains first and second order derivatives with respect to $y$ and $z$, then $\mathcal{L}_{1}^{S} P_{0}^{S, \epsilon}=0$. The equation becomes then $\mathcal{L}_{0}^{S} P_{1}^{S, \epsilon}=0$. The equation is satisfied if $P_{1}^{S, \epsilon}=P_{1}^{S, \epsilon}\left(t, S_{t}\right)$ independent of $Y_{t}$ and $Z_{t}$.

Consequently, $P_{0}^{S, \epsilon}$ and $P_{1}^{S, \epsilon}$ are independent of $Y_{t}$ and $Z_{t}$, and we have:

$$
\mathcal{L}_{0}^{S} P_{0}^{S, \epsilon}=\mathcal{L}_{1}^{S} P_{0}^{S, \epsilon}=\mathcal{L}_{0}^{S} P_{1}^{S, \epsilon}=\mathcal{L}_{1}^{S} P_{1}^{S, \epsilon}=0 .
$$

Given that $\mathcal{L}_{1}^{S} P_{1}^{S, \epsilon}=0$, the term of order 0 in $\sqrt{\epsilon}$ becomes:

$$
\mathcal{L}_{2}^{S} P_{0}^{S, \epsilon}+\mathcal{L}_{0}^{S} P_{2}^{S, \epsilon}=0
$$

This is a Poisson equation for $P_{2}^{S, \epsilon}$ with respect to $\mathcal{L}_{0}^{S}$. The solvability condition for this equation is:

$$
<\mathcal{L}_{2}^{S} P_{0}^{S, \epsilon}>_{1,2}=<\mathcal{L}_{2}^{S}>_{1,2} P_{0}^{S, \epsilon}=0
$$

We can notice that the average $<\mathcal{L}_{2}^{S}>$ of the generator $\mathcal{L}_{2}^{S}$ is equal to :

$$
<\mathcal{L}_{2}^{S}>_{1,2}=\frac{\partial}{\partial t}+r\left(\frac{\partial}{\partial S_{t}} S_{t}-.\right)+\frac{1}{2} \frac{\partial^{2}}{\partial S_{t}^{2}} S_{t}^{2}<\beta^{2} f_{1}^{2}(y)+f_{2}^{2}(z)>_{1,2} .
$$

Then, we can deduce that: $<\mathcal{L}_{2}^{S}>_{1,2}=\mathcal{L}_{B S}^{S}\left(\bar{\sigma}_{S}\right)$ where $\bar{\sigma}_{S}^{2}=\beta^{2}<f_{1}^{2}>_{1,2}+<f_{2}^{2}>_{1,2}$. 
Consequently, $P_{0}^{S, \epsilon}$ is the solution of the following problem :

$$
\begin{aligned}
\mathcal{L}_{B S}\left(\bar{\sigma}_{S}\right) P_{0}^{S, \epsilon} & =0, \\
P_{0}^{S, \epsilon}\left(T, S_{T}\right) & =h\left(S_{T}\right) .
\end{aligned}
$$

We deduce that $P_{0}^{S, \epsilon}$ is the Black-Scholes price of the option with implied volatility equal to $\bar{\sigma}_{S}$, meaning that:

$$
P_{0}^{S, \epsilon}=P_{B S}^{S}\left(t, S_{t}, \bar{\sigma}_{S}\right) .
$$

The term of order 1 in $\sqrt{\epsilon}$ is a Poisson equation for $P_{3}^{S, \epsilon}$ with respect to $\mathcal{L}_{0}^{S}$. The solvability condition for this equation is:

$$
<\mathcal{L}_{2}^{S}>_{1,2} P_{1}^{S, \epsilon}=-<\mathcal{L}_{1}^{S} P_{2}^{S, \epsilon}>_{1,2}=<\mathcal{L}_{1}^{S}\left(\mathcal{L}_{0}^{S}\right)^{-1}\left(\mathcal{L}_{2}^{S}-<\mathcal{L}_{2}^{S}>_{1,2}\right)>P_{0}^{S, \epsilon} .
$$

$P_{1}^{S, \epsilon}$ is the solution of the last equation with terminal condition $P_{1}^{S, \epsilon}\left(T, S_{t}\right)=0$.

Given that $f_{1}$ is independent of $z$ and $f_{2}$ is independent of $y$, we have: $<f_{1}^{2}>_{1,2}=<f_{1}^{2}>_{1}$ and $<f_{2}^{2}>_{1,2}=<f_{2}^{2}>_{2}$. We recall here that $\phi_{I}$, the solution of (3.6), doesn't depend on $z$, we deduce that:

$$
\mathcal{L}_{0}^{S} \phi_{I}(y)=\mathcal{L}_{0}^{I} \phi_{I}(y)=f_{1}^{2}(y)-<f_{1}^{2}>_{1,2} .
$$

Let $\phi_{\text {Idios }}$ the solution of the following equation:

$$
\mathcal{L}_{0}^{S} \phi_{\text {Idios }}(z)=f_{2}^{2}(z)-<f_{2}^{2}>_{1,2} .
$$

Given that $\phi_{\text {Idios }}$ doesn't depend on $y$, we obtain that:

$$
\mathcal{L}_{0}^{S}\left(\beta^{2} \phi_{I}(y)+\phi_{\text {Idios }}(z)\right)=\beta^{2}\left(f_{1}^{2}(y)-<f_{1}^{2}>_{1,2}\right)+\left(f_{2}^{2}(z)-<f_{2}^{2}>_{1,2}\right) .
$$

Then we deduce that:

$$
\mathcal{L}_{1}^{S}\left(\mathcal{L}_{0}^{S}\right)^{-1}\left(\mathcal{L}_{2}^{S}-<\mathcal{L}_{2}^{S}>_{1,2}\right)=\left(\beta^{2} \mathcal{L}_{1}^{S} \phi_{I}(y)+\mathcal{L}_{1}^{S} \phi_{\text {Idios }}(z)\right) \frac{1}{2} S_{t}^{2} \frac{\partial^{2}}{\partial S_{t}^{2}} .
$$

By developing the right term in the previous equation, we obtain:

$$
\begin{aligned}
\frac{1}{2}\left(\beta^{2}<\mathcal{L}_{1}^{S} \phi_{I}(y)>_{1,2}+<\mathcal{L}_{1}^{S} \phi_{\text {Idios }}(z)>_{1,2}\right) & =\left(\frac{\beta^{3} \nu_{Y} \rho_{Y}}{\sqrt{2}}<\phi_{I}^{\prime} f_{1}>_{1,2}+\frac{\rho_{Z} \nu_{Z} \sqrt{\alpha}}{\sqrt{2}}<\phi_{\text {Idios }}^{\prime} f_{2}>_{1,2}\right) S_{t} \frac{\partial}{\partial S_{t}} \\
& -\left(\frac{\beta^{2} \nu_{Y}}{\sqrt{2}}<\phi_{I}^{\prime} \chi_{1}>_{1,2}+\frac{\nu_{Z} \sqrt{\alpha}}{\sqrt{2}}<\phi_{\text {Idios }}^{\prime} \chi_{2}>_{1,2}\right)
\end{aligned}
$$

In order to simplify the notations, we define the quantities $V_{2}^{S, \epsilon}$ and $V_{3}^{S, \epsilon}$ :

$$
\begin{aligned}
V_{3}^{S, \epsilon} & =\frac{\sqrt{\epsilon}}{\sqrt{2}}\left(\beta^{3} \nu_{Y} \rho_{Y}<\phi_{I}^{\prime} f_{1}>+\rho_{Z} \nu_{Z} \sqrt{\alpha}<\phi_{\text {Idios }}^{\prime} f_{2}>\right), \\
V_{2}^{S, \epsilon} & =-\frac{\sqrt{\epsilon}}{\sqrt{2}}\left(\beta^{2} \nu_{Y}<\phi_{I}^{\prime} \chi_{1}>+\nu_{Z} \sqrt{\alpha}<\phi_{\text {Idios }}^{\prime} \chi_{2}>\right) .
\end{aligned}
$$


The equation (3.17) becomes then:

$$
<\mathcal{L}_{2}^{S}>_{1,2} \sqrt{\epsilon} P_{1}^{S, \epsilon}=V_{2}^{S, \epsilon} S_{t}^{2} \frac{\partial^{2} P_{0}^{S, \epsilon}}{\partial S_{t}^{2}}+V_{3}^{S, \epsilon} S_{t} \frac{\partial}{\partial S_{t}}\left(S_{t}^{2} \frac{\partial^{2} P_{0}^{S, \epsilon}}{\partial S_{t}^{2}}\right) .
$$

Consequently, $P_{1}^{S, \epsilon}$ is the solution of $(3.19)$ with the final condition $P_{1}^{S, \epsilon}\left(T, S_{T}\right)=0$. Given that $<\mathcal{L}_{2}^{S}>_{1,2}=\mathcal{L}_{B S}^{S}\left(\bar{\sigma}_{S}\right)$ commits with the operators $\mathcal{D}_{1, S}=S_{t} \frac{\partial}{\partial S_{t}}$ and $\mathcal{D}_{2, S}=S_{t}^{2} \frac{\partial^{2}}{\partial S_{t}^{2}}$, and that $<\mathcal{L}_{2}^{S}>_{1,2} P_{0}^{S, \epsilon}=0$, the solution to the last problem can be given explicitly by :

$$
\sqrt{\epsilon} P_{1}^{S, \epsilon}=-(T-t)\left(V_{2}^{S, \epsilon} S_{t}^{2} \frac{\partial^{2} P_{0}^{S, \epsilon}}{\partial S_{t}^{2}}+V_{3}^{S, \epsilon} S_{t} \frac{\partial}{\partial S_{t}}\left(S_{t}^{2} \frac{\partial^{2} P_{0}^{S, \epsilon}}{\partial S_{t}^{2}}\right)\right) .
$$

In order to check the validity of the solution, we can easily make the following verification:

$$
\begin{aligned}
<\mathcal{L}_{2}^{S}>_{1,2} \sqrt{\epsilon} P_{1}^{S, \epsilon} & =\left(V_{2}^{S, \epsilon} \mathcal{D}_{2, S} P_{0}^{S, \epsilon}+V_{3}^{S, \epsilon} \mathcal{D}_{1, S} \mathcal{D}_{2, S} P_{0}^{S, \epsilon}\right)<\mathcal{L}_{2}^{S}>_{1,2}((t-T)) \\
& -(T-t)\left(V_{2}^{S, \epsilon} \mathcal{D}_{2, S}<\mathcal{L}_{2}^{S}>_{1,2}\left(P_{0}^{S, \epsilon}\right)+V_{3}^{S, \epsilon} \mathcal{D}_{1, S} \mathcal{D}_{2, S}<\mathcal{L}_{2}^{S}>_{1,2}\left(P_{0}^{S, \epsilon}\right)\right) \\
& =V_{2}^{S, \epsilon} \mathcal{D}_{2, S} P_{0}^{S, \epsilon}+V_{3}^{S, \epsilon} \mathcal{D}_{1, S} \mathcal{D}_{2, S} P_{0}^{S, \epsilon}
\end{aligned}
$$

By neglecting terms of order higher or equal to 1 in $\sqrt{\epsilon}$, we can approximate the stock option's price by $\left(P_{0}^{S, \epsilon}+\sqrt{\epsilon} P_{1}^{S, \epsilon}\right)$. In order to reduce the number of the parameters in the approximation, we prove that:

$$
P_{0}^{S, \epsilon}+\sqrt{\epsilon} P_{1}^{S, \epsilon}=\tilde{P}_{0}^{S, \epsilon}+\sqrt{\epsilon} \tilde{P}_{1}^{S, \epsilon}+o(\epsilon),
$$

where $\tilde{P}_{0}^{S, \epsilon}$ is defined as:

$$
\begin{aligned}
\mathcal{L}_{B S}\left(\bar{\sigma}_{S}^{*}\right) \tilde{P}_{0} & =0, \\
\tilde{P}_{0}^{S, \epsilon}\left(T, S_{T}\right) & =\left(S_{T}-K_{S}\right)^{+},
\end{aligned}
$$

and $\mathcal{L}_{B S}\left(\bar{\sigma}_{S}^{*}\right)$ is the Black-Scholes differential operator with volatility $\bar{\sigma}_{S}^{*}$ such that:

$$
\left(\bar{\sigma}_{S}^{*}\right)^{2}=\bar{\sigma}_{S}^{2}-2 V_{2}^{S, \epsilon}
$$

which gives $\tilde{P}_{0}^{S, \epsilon}=P_{B S}\left(t, S_{t}, \bar{\sigma}_{S}^{*}\right)$. On the other hand, $\tilde{P}_{1}^{S, \epsilon}$ is defined as:

$$
\begin{aligned}
\mathcal{L}_{B S}\left(\bar{\sigma}_{S}^{*}\right) \sqrt{\epsilon} \tilde{P}_{1}^{S, \epsilon} & =V_{3}^{S, \epsilon} S_{t} \frac{\partial}{\partial S_{t}}\left(S_{t}^{2} \frac{\partial^{2}}{\partial S_{t}^{2}} \tilde{P}_{0}^{S, \epsilon}\right), \\
\tilde{P}_{1}^{S, \epsilon}\left(T, S_{T}\right) & =0 .
\end{aligned}
$$

We can notice that:

$$
\mathcal{L}_{B S}\left(\bar{\sigma}_{S}^{*}\right)=\mathcal{L}_{B S}\left(\bar{\sigma}_{S}\right)-V_{2}^{S, \epsilon} S_{t}^{2} \frac{\partial^{2}}{\partial S_{t}^{2}}
$$

We start by studying the term $\left(P_{0}^{S, \epsilon}-\tilde{P}_{0}^{S, \epsilon}\right)$. Using 3.21 , we can then easily prove that:

$$
\begin{aligned}
\mathcal{L}_{B S}\left(\bar{\sigma}_{S}\right)\left(P_{0}^{S, \epsilon}-\tilde{P}_{0}^{S, \epsilon}\right) & =-V_{2}^{S, \epsilon} S_{t}^{2} \frac{\partial^{2} \tilde{P}_{0}^{S, \epsilon}}{\partial S_{t}^{2}}, \\
\left(P_{0}^{S, \epsilon}-\tilde{P}_{0}^{S, \epsilon}\right)\left(T, S_{T}\right) & =0 .
\end{aligned}
$$


Since the source term is $O(\sqrt{\epsilon})$ because of $V_{2}^{S, \epsilon}$, then the difference term $\left(P_{0}^{S, \epsilon}-\tilde{P}_{0}^{S, \epsilon}\right)$ is also $O(\sqrt{\epsilon})$. Next, we write:

$$
\left|P^{S, \epsilon}-\left(\tilde{P}_{0}^{S, \epsilon}+\sqrt{\epsilon} \tilde{P}_{1}^{S, \epsilon}\right)\right| \leq\left|P^{S, \epsilon}-\left(P_{0}^{S, \epsilon}+\sqrt{\epsilon} P_{1}^{S, \epsilon}\right)\right|+\left|\left(P_{0}^{S, \epsilon}+\sqrt{\epsilon} P_{1}^{S, \epsilon}\right)-\left(\tilde{P}_{0}^{S, \epsilon}+\sqrt{\epsilon} \tilde{P}_{1}^{S, \epsilon}\right)\right|
$$

The first term $\left|P^{S, \epsilon, \delta}-\left(P_{0}^{S, \epsilon}+\sqrt{\epsilon} P_{1}^{S, \epsilon}\right)\right|$ is already $o(\epsilon)$. We focus then on the second term. To simplify the notations, we introduce the error term:

$$
\mathcal{R}=\left(P_{0}^{S, \epsilon}+\sqrt{\epsilon} P_{1}^{S, \epsilon}\right)-\left(\tilde{P}_{0}^{S, \epsilon}+\sqrt{\epsilon} \tilde{P}_{1}^{S, \epsilon}\right),
$$

and the differential operators:

$$
\begin{aligned}
\mathcal{H}_{\epsilon} & =V_{2}^{S, \epsilon} \mathcal{D}_{2, S}+V_{3}^{S, \epsilon} \mathcal{D}_{1, S} \mathcal{D}_{2, S} \\
\mathcal{H}_{\epsilon}^{*} & =V_{3}^{S, \epsilon} \mathcal{D}_{1, S} \mathcal{D}_{2, S}
\end{aligned}
$$

We can then compute the term:

$$
\begin{aligned}
\mathcal{L}_{B S}\left(\bar{\sigma}_{S}\right) \mathcal{R} & =\mathcal{L}_{B S}\left(\bar{\sigma}_{S}\right)\left(\left(P_{0}^{S, \epsilon}+\sqrt{\epsilon} P_{1}^{S, \epsilon}\right)-\left(\tilde{P}_{0}^{S, \epsilon}+\sqrt{\epsilon} \tilde{P}_{1}^{S, \epsilon}\right)\right), \\
& =\mathcal{H}_{\epsilon} P_{0}^{S, \epsilon}-\left(\mathcal{L}_{B S}\left(\bar{\sigma}_{S}^{*}\right)+V_{2}^{S, \epsilon} \mathcal{D}_{2, S}\right)\left(\tilde{P}_{0}^{S, \epsilon}+\sqrt{\epsilon} \tilde{P}_{1}^{S, \epsilon}\right), \\
& =\mathcal{H}_{\epsilon} P_{0}^{S, \epsilon}-\mathcal{H}_{\epsilon}^{*} \tilde{P}_{0}^{S, \epsilon}-V_{2}^{S, \epsilon} \mathcal{D}_{2, S}\left(\tilde{P}_{0}^{S, \epsilon}+\sqrt{\epsilon} \tilde{P}_{1}^{S, \epsilon}\right), \\
& =\mathcal{H}_{\epsilon}^{*}\left(P_{0}^{S, \epsilon}-\tilde{P}_{0}^{S, \epsilon}\right)-V_{2}^{S, \epsilon} \mathcal{D}_{2, S}\left(\tilde{P}_{0}^{S, \epsilon}-P_{0}^{S, \epsilon}+\sqrt{\epsilon} \tilde{P}_{1}^{S, \epsilon}\right) .
\end{aligned}
$$

Knowing that:

- $\left(P_{0}^{S, \epsilon}-\tilde{P}_{0}^{S, \epsilon}\right)$ is $O(\sqrt{\epsilon})$.

- $\mathcal{H}_{\epsilon}^{*}$ is $O(\sqrt{\epsilon})$

- $V_{2}^{S, \epsilon} \mathcal{D}_{2, S}$ is $O(\sqrt{\epsilon})$

- $\sqrt{\epsilon} \tilde{P}_{1}^{S, \epsilon}$ is $O(\sqrt{\epsilon})$

and additionally $\mathcal{R}(T)=0$, then we can obtain that:

$$
\mathcal{R}=O(\epsilon) .
$$

This concludes the derivation of the following result:

$$
P_{0}^{S, \epsilon}+\sqrt{\epsilon} P_{1}^{S, \epsilon}=\tilde{P}_{0}^{S, \epsilon}+\sqrt{\epsilon} \tilde{P}_{1}^{S, \epsilon}+O(\epsilon) .
$$

So up to order 1 in $\sqrt{\epsilon}$, we can approximate the option price by $\tilde{P}^{S, \epsilon}$ which is defined as:

$$
\tilde{P}^{S, \epsilon}=\tilde{P}_{0}^{S, \epsilon}+\sqrt{\epsilon} \tilde{P}_{1}^{S, \epsilon} .
$$

Fouque and $a l$ proved that the estimation error obtained, when approximating $P^{S, \epsilon}$ by $\tilde{P}^{S, \epsilon}$, is at order 1 in $\epsilon$. The reader can refer to Appendix 2 for a brief review of the proof. 


\subsection{Calibration of implied beta using options prices}

We have already given an approximation for the price of an european option on the index in (3.1) and an approximation for the price of an european option on the stock in (3.7). In this section, an estimator of the parameter $\beta$ using options prices is provided.

\subsubsection{Approximation formula of the implied volatility smile}

We derive an approximation of the smile of implied volatility for both the stock and the index in function of model parameters. As suggested in [6], we perform an expansion of the implied volatility in powers of $\sqrt{\epsilon}$. This enables us to approximate the shape of the implied volatility surfaces for both the stock and the index and to obtain the following results:

Proposition 3.2 The implied volatility of an european call on the index with strike $K_{I}$ and maturity $T$ can be approximated, at order 1 in $\sqrt{\epsilon}$, by $\Sigma_{I}\left(K_{I}, T\right)$ which can be given as:

$$
\Sigma_{I}\left(K_{I}, T\right)=b_{I}+a_{I} \frac{\ln \left(\frac{F_{I}}{K_{I}}\right)}{T}
$$

where $b_{I}=\bar{\sigma}_{I}^{*}-\frac{V_{3}^{I, \epsilon}}{2 \bar{\sigma}_{I}^{*}}, a_{I}=\frac{V_{3}^{I, \epsilon}}{\left(\bar{\sigma}_{I}^{*}\right)^{3}}$ and $F_{I}=I_{t} e^{r(T-t)}$.

On the other hand, the implied volatility of an european call on the stock with strike $K_{S}$ and maturity $T$ can be approximated, at order 1 in $\sqrt{\epsilon}$, by $\Sigma_{S}\left(K_{S}, T\right)$ which is equal to:

$$
\Sigma_{S}(K, T)=b_{S}+a_{S} \frac{\ln \left(\frac{F_{S}}{K_{S}}\right)}{T}
$$

where $b_{S}=\bar{\sigma}_{S}^{*}-\frac{V_{3}^{S, \epsilon}}{2 \bar{\sigma}_{S}^{*}}, a_{S}=\frac{V_{3}^{S, \epsilon}}{\left(\bar{\sigma}_{S}^{*}\right)^{3}}$ and $F_{S}=S_{t} e^{r(T-t)}$.

A derivation of this result is included here for the purpose of making this paper as selfcontained as possible.

\section{Proof:}

We use here $A$ to denote either the stock $S$ or the index $I$. We have :

$$
P^{A, \epsilon}=\tilde{P}_{0}^{A, \epsilon}-(T-t) V_{3}^{A, \epsilon} A_{t} \frac{\partial}{\partial A_{t}}\left(A_{t}^{2} \frac{\partial^{2} \tilde{P}_{0}^{A, \epsilon}}{\partial A_{t}^{2}}\right)+o(\epsilon),
$$

where $\tilde{P}_{0}^{A, \epsilon}$ is defined as:

$$
\tilde{P}_{0}^{A, \epsilon}=P_{B S}\left(t, A_{t}, \bar{\sigma}_{A}^{*}\right)
$$

$P^{A, \epsilon}$ could represent the price of the option on the index $P^{I, \epsilon}$ if $A=I$ or the price of the option on the stock $P^{S, \epsilon}$ in the case where $A=S$. 
Let $I_{A}$ be the implied volatility associated to the asset's option price $P^{A, \epsilon}$, then we have $P^{A, \epsilon}=P_{B S}\left(t, A_{t}, I_{A}\left(K_{A}, T\right)\right)$. We can do an expansion of $I_{A}(K, T)$ around $\bar{\sigma}_{A}^{*}$ in powers of $\sqrt{\epsilon}:$

$$
I_{A}\left(K_{A}, T\right)=\bar{\sigma}_{A}^{*}+\sqrt{\epsilon} I_{1}\left(K_{A}, T\right)+O(\epsilon)
$$

We have then:

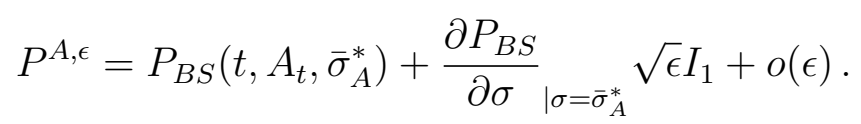

If we combine the equations 3.21 and 3.22 , we get:

$$
\frac{\partial P_{B S}\left(t, A_{t}, \bar{\sigma}_{A}^{*}\right)}{\partial \sigma}{ }_{\mid \sigma=\bar{\sigma}_{A}^{*}} \sqrt{\epsilon} I_{1}(K, T)=-(T-t) V_{3}^{A, \epsilon} A_{t} \frac{\partial}{\partial A_{t}}\left(A_{t}^{2} \frac{\partial^{2} \tilde{P}_{0}}{\partial A_{t}^{2}}\right) .
$$

By performing simple computations on the derivatives of the Black-Scholes price, we can obtain that:

$$
\mathcal{D}_{2, A} \tilde{P}_{0}=\frac{1}{\bar{\sigma}_{A}^{*}(T-t)} \frac{\partial P_{B S}}{\partial \bar{\sigma}_{A}^{*}}\left(t, A_{t}, \bar{\sigma}_{A}^{*}\right)
$$

We apply then the operator $\mathcal{D}_{1, A}$ to the last equation:

$$
\mathcal{D}_{1, A} \mathcal{D}_{2, A} \tilde{P}_{0}=\frac{A_{t}}{\bar{\sigma}_{A}^{*}(T-t)} \frac{\partial^{2} P_{B S}}{\partial A_{t} \partial \bar{\sigma}_{A}^{*}}\left(t, A_{t}, \bar{\sigma}_{A}^{*}\right) .
$$

Using closed-form formulas of Black-Scholes greeks, we can write that:

$$
A_{t} \frac{\partial^{2} P_{B S}}{\partial A_{t} \partial \bar{\sigma}_{A}^{*}}\left(t, A_{t}, \bar{\sigma}_{A}^{*}\right)=-\frac{d_{2}}{\bar{\sigma}_{A}^{*} \sqrt{T-t}} \frac{\partial P_{B S}}{\partial \bar{\sigma}_{A}^{*}}\left(t, A_{t}, \bar{\sigma}_{A}^{*}\right) .
$$

The equation 3.23 can be written as:

$$
\sqrt{\epsilon} I_{1}\left(K_{A}, T\right)=-\frac{V_{3}^{A, \epsilon}}{\bar{\sigma}_{A}^{*}} \frac{A_{t} \frac{\partial^{2} \tilde{P}_{0}}{\partial A_{t} \partial \bar{\sigma}_{A}^{*}}}{\frac{\partial \tilde{P}_{0}}{\partial \bar{\sigma}_{A}^{*}}}
$$

Then, it is straightforward that:

$$
\sqrt{\epsilon} I_{1}\left(K_{A}, T\right)=\frac{V_{3}^{A, \epsilon} d_{2}\left(K_{A}, T\right)}{\left(\bar{\sigma}_{A}^{*}\right)^{2} \sqrt{T-t}},
$$

where:

$$
d_{2}\left(K_{A}, T\right)=\frac{\log \left(\frac{A_{t} e^{r(T-t)}}{K_{A}}\right)-\frac{\left(\bar{\sigma}_{A}^{*}\right)^{2}}{2}(T-t)}{\bar{\sigma}_{A}^{*} \sqrt{T-t}}
$$


We can then approximate the implied volatility by the following formula:

$$
I_{A}\left(K_{A}, T\right)=\bar{\sigma}_{A}^{*}-\frac{V_{3}^{A, \epsilon}}{2 \bar{\sigma}_{A}^{*}}+\frac{V_{3}^{A, \epsilon}}{\left(\bar{\sigma}_{A}^{*}\right)^{3}} \frac{\ln \left(\frac{F_{A}(T)}{K_{A}}\right)}{T-t} .
$$

Then, we obtain the following smile approximation expression:

$$
I_{A}\left(K_{A}, T\right)=b_{A}+a_{A} \frac{\ln \left(\frac{F_{A}(T)}{K_{A}}\right)}{T-t},
$$

with:

$$
\begin{aligned}
& b_{A}=\bar{\sigma}_{A}^{*}-\frac{V_{3}^{A, \epsilon}}{2 \bar{\sigma}_{A}^{*}}, \\
& a_{A}=\frac{V_{3}^{A, \epsilon}}{\left(\bar{\sigma}_{A}^{*}\right)^{3}} .
\end{aligned}
$$

\subsubsection{Comparison with the model with constant idiosyncratic volatility}

We use the approximations given previously in order to estimate the parameter $\beta$. Based on the definitions of $V_{3}^{S, \epsilon}$ and $V_{3}^{I, \epsilon}$, we can write that:

$$
\begin{aligned}
V_{3}^{S, \epsilon} & =\beta^{3} V_{3}^{I, \epsilon}+\frac{\sqrt{\epsilon}}{\sqrt{2}} \rho_{Z} \nu_{Z} \sqrt{\alpha}<\phi_{\text {Idios }}^{\prime} f_{2}>, \\
\frac{V_{3}^{S, \epsilon}}{V_{3}^{I, \epsilon}} & =\beta^{3}+\frac{\rho_{Z} \nu_{Z} \sqrt{\alpha}}{\rho_{Y} \nu_{Y}} \frac{\phi_{\text {Idio }}^{\prime} f_{2}>}{<\phi_{I}^{\prime} f_{1}>} .
\end{aligned}
$$

The estimator $\hat{\beta}$ proposed in $[6]$ and introduced also in 2.1 verifies:

$$
\hat{\beta}^{3}=\frac{V_{3}^{S, \epsilon}}{V_{3}^{I, \epsilon}}=\beta^{3}+\frac{\rho_{Z} \nu_{Z} \sqrt{\alpha}}{\rho_{Y} \nu_{Y}} \frac{<\phi_{\text {Idio }}^{\prime} f_{2}>}{<\phi_{I}^{\prime} f_{1}>} .
$$

We deduce that in the case where the idiosyncratic volatility of the stock is stochastic, $\hat{\beta}$ is a biased estimator of the parameter $\beta$.

\subsubsection{Alternative method for the estimation}

We recall here that:

$$
\bar{\sigma}_{S}^{2}=\beta^{2} \bar{\sigma}_{I}^{2}+<f_{2}^{2}>_{1,2} .
$$

We can then write that:

$$
\beta^{2}=\frac{\left(\bar{\sigma}_{S}^{*}\right)^{2}-<f_{2}^{2}>_{1,2}}{\left(\bar{\sigma}_{I}^{*}\right)^{2}+2 V_{2}^{I, \epsilon}}+\frac{2 V_{2}^{S, \epsilon}}{\left(\bar{\sigma}_{I}^{*}\right)^{2}+2 V_{2}^{I, \epsilon}} .
$$


Given the values of $V_{2}^{S, \epsilon}$ and $V_{2}^{I, \epsilon}$, we can then write $\beta$ as following:

$$
\beta=\sqrt{\frac{\left(\bar{\sigma}_{S}^{*}\right)^{2}-<f_{2}^{2}>_{1,2}}{\left(\bar{\sigma}_{I}^{*}\right)^{2}+2 V_{2}^{I, \epsilon}}+\frac{2 V_{2}^{S, \epsilon}}{\left(\bar{\sigma}_{I}^{*}\right)^{2}+2 V_{2}^{I, \epsilon}}} .
$$

Using the smile approximation formula (3.24), we can write, for the asset $A$ which denotes either the stock $S$ or the index $I$, the following relation:

$$
b_{A}=\bar{\sigma}_{A}^{*}-\frac{1}{2} a_{A}\left(\bar{\sigma}_{A}^{*}\right)^{2} .
$$

This second order equation in $\bar{\sigma}_{A}^{*}$ has two admissible solutions $x_{1}$ and $x_{2}$ :

$$
\begin{aligned}
& x_{1}=\frac{1-\sqrt{1-2 a_{A} b_{A}}}{a_{A}}, \\
& x_{2}=\frac{1+\sqrt{1-2 a_{A} b_{A}}}{a_{A}} .
\end{aligned}
$$

Since $V_{2}^{A, \epsilon}$ and $V_{3}^{A, \epsilon}$ are of order 1 in $\sqrt{\epsilon}$, then $a_{A}$ is of order 1 in $\sqrt{\epsilon}$ and $b_{A}=\bar{\sigma}_{A}^{*}+o(\sqrt{\epsilon})$. We can then deduce that the appropriate solution is $x_{1}$. Consequently, we have:

$$
\begin{aligned}
& \bar{\sigma}_{S}^{*}=\frac{1-\sqrt{1-2 a_{S} b_{S}}}{a_{S}}, \\
& \bar{\sigma}_{I}^{*}=\frac{1-\sqrt{1-2 a_{I} b_{I}}}{a_{I}} .
\end{aligned}
$$

Then, we can approximate $\beta$ by $\tilde{\beta}$ which is given by:

$$
\tilde{\beta}=\sqrt{\frac{\left(\frac{1-\sqrt{1-2 a_{S} b_{S}}}{a_{S}}\right)^{2}-<f_{2}^{2}>_{1,2}}{\left(\frac{1-\sqrt{1-2 a_{I} b_{I}}}{a_{I}}\right)^{2}+2 V_{2}^{I, \epsilon}}+\frac{2 V_{2}^{S, \epsilon}}{\left(\frac{1-\sqrt{1-2 a_{I} b_{I}}}{a_{I}}\right)^{2}+2 V_{2}^{I, \epsilon}}} .
$$

In order to compute $\tilde{\beta}$, we follow the methodology given below:

- We approximate $\bar{\sigma}_{I}$ and $\bar{\sigma}_{S}$ by the historical volatilities of the market index and the stock respectively using underlying log-returns.

- We calibrate an affine function in the composite variable called "log-moneyness-tomaturity ratio" to the implied volatility surfaces of the index and the stock across different strikes and maturities. We get then the estimated slopes $a_{S}, a_{I}$ and the intercepts $b_{S}, b_{I}$.

- From the estimated slope $a_{S}$, the intercept $b_{S}$ and the effective volatility $\bar{\sigma}_{S}$, we calculate the following quantities:

$$
\begin{aligned}
& -\bar{\sigma}_{S}^{*}=\frac{1-\sqrt{1-2 a_{S} b_{S}}}{a_{S}} \\
& -V_{2}^{S, \epsilon}=\frac{\bar{\sigma}_{S}^{2}-\left(\bar{\sigma}_{S}^{*}\right)^{2}}{2}
\end{aligned}
$$




$$
-V_{3}^{S, \epsilon}=a_{S}\left(\bar{\sigma}_{S}^{*}\right)^{3}
$$

- Likewise, from the estimated slope $a_{I}$, the intercept $b_{I}$ and the effective volatility $\bar{\sigma}_{I}$, we calculate the following quantities:

$$
\begin{aligned}
& -\bar{\sigma}_{I}^{*}=\frac{1-\sqrt{1-2 a_{I} b_{I}}}{a_{I}} \\
& -V_{2}^{I, \epsilon}=\frac{\bar{\sigma}_{I}^{2}-\left(\bar{\sigma}_{I}^{*}\right)^{2}}{2} \\
& -V_{3}^{I, \epsilon}=a_{I}\left(\bar{\sigma}_{I}^{*}\right)^{3}
\end{aligned}
$$

- We use the time series of the log-returns of the stock $(S)$ and the index $(I)$ in order to deduce the time serie of the idiosyncratic volatility $\left(f\left(Z_{t}\right)\right)_{t}$. We calibrate the parameters $m_{Z}, \nu_{Z}$ and $\rho_{Z}$ on the time serie of $\left(Z_{t}\right)_{t}$. For this purpose, we use the maximum likelihood estimation method suggested in [8]. We evaluate then the quantity $<f_{2}^{2}>_{1,2}$. If we consider the case where $f_{2}$ denotes the exponential function, then we have:

$$
<f_{2}^{2}>_{1,2}=\int_{-\infty}^{+\infty} e^{2 z} \frac{1}{\sqrt{2 \pi}} \exp \left(-\frac{\left(z-m_{Z}\right)^{2}}{2 \nu_{Z}^{2}}\right) d z=e^{2 m_{Z}+2 \nu_{Z}^{2}}
$$

- We compute finally the value of $\tilde{\beta}$ using 3.26 .

\section{Empirical study}

\subsection{Numerical illustrations}

In this section, we will estimate the parameter $\beta$ according to the methods (2.1) and (3.26) using real data. For this purpose, we use options prices whose maturities are equal to $T=0.5$ and whose moneyness range from $80 \%$ to $120 \%$.

Furthermore, we introduce a third estimator, the historical beta $\beta_{H}$, which is used traditionally as an estimator of the parameter $\beta . \beta_{H}$ is a backward measure of the realization of the parameter $\beta$. It is obtained, at a given date $t$, as the slope of the linear regression of the stock daily returns on the index daily returns between $t-L$ and $t$. We intentionally fixed $L$, the length of the backward window on which we perform the linear regression, to be equal to the maturity of the options we use to compute $\tilde{\beta}$ and $\hat{\beta}$. We have then $L=T=0.5$

We use here spot and options data on a sample of SPX 500 stocks and ETF and the SPX 500 market index. The sample includes Financial Select Sector (XLF), Energy Select Sector (XLE), Materials Select Sector (XLB), Technology Select Sector (XLK), Industrial Select Sector (XLI), Goldman Sachs (GS), Microsoft (MSFT), General Electric (GE), Google (GOOG), IBM (IBM), JP Morgan (JPM), Cisco Systems (CSCO), Bank Of America (BAC), Intel Corporation (INTC), Chevron Corporation (CVX), Caterpillar Inc(CAT), Apple Inc (AAPL), Alcoa Inc (AA). The data used for this empirical study range between 01/01/2008 and $31 / 12 / 2012$. 
For every date $t$ of the sample, we compute the estimators $\hat{\beta}(t)$ and $\tilde{\beta}(t)$, according to (2.1) and 3.26 respectively, by using options prices with maturity $T=6 M$ and moneyness $M$ between $80 \%$ and $120 \%$. We use also the linear regression method on a backward window with size $L$ equal to $T$ in order to compute $\beta_{H}(t)$.

The graphs below represent the time series $(\hat{\beta}(t))_{t_{0} \leq t \leq t_{N}},(\tilde{\beta}(t))_{t_{0} \leq t \leq t_{N}}$ and $\left(\beta_{H}(t)\right)_{t_{0} \leq t \leq t_{N}}$, for $t_{0}=01 / 01 / 2008$ and $t_{N}=31 / 12 / 2012$. 


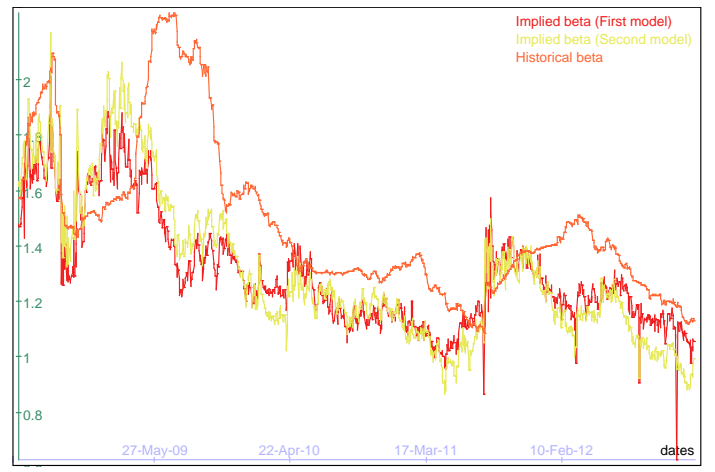

(a) XLF vs SPX

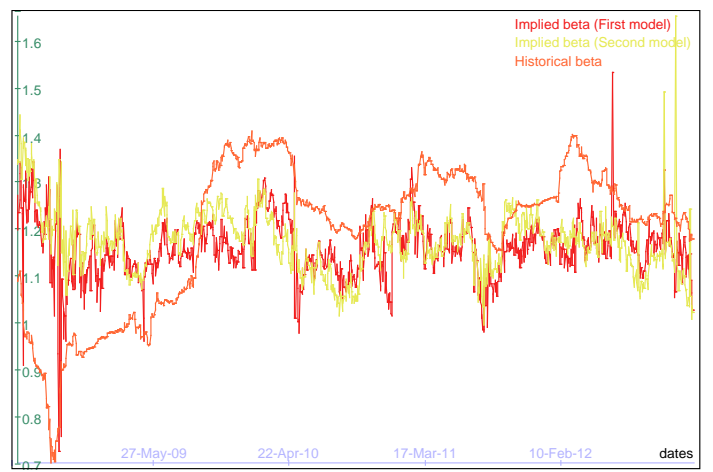

(c) XLB vs SPX

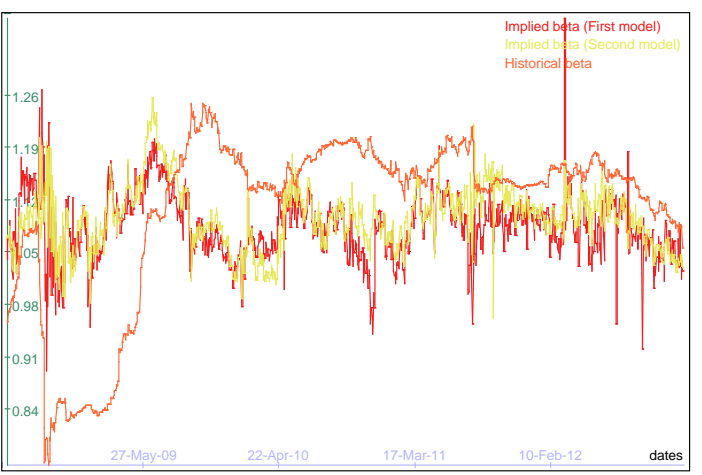

(e) XLI vs SPX

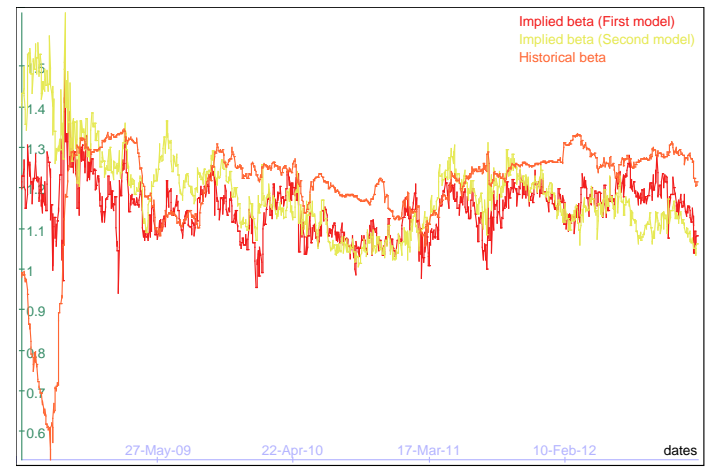

(b) XLE vs SPX

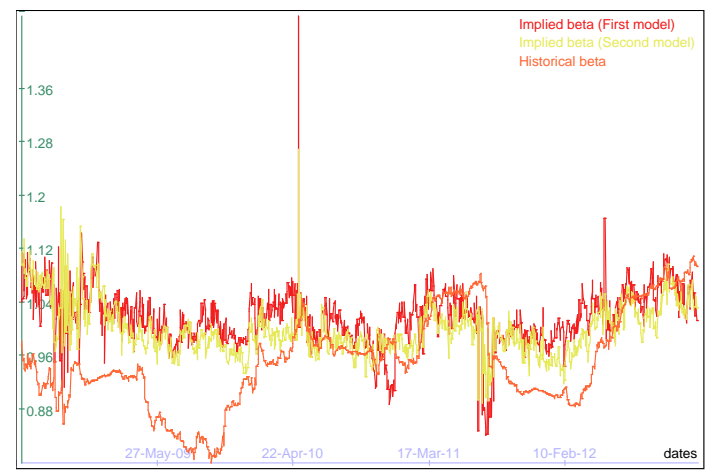

(d) XLK vs SPX

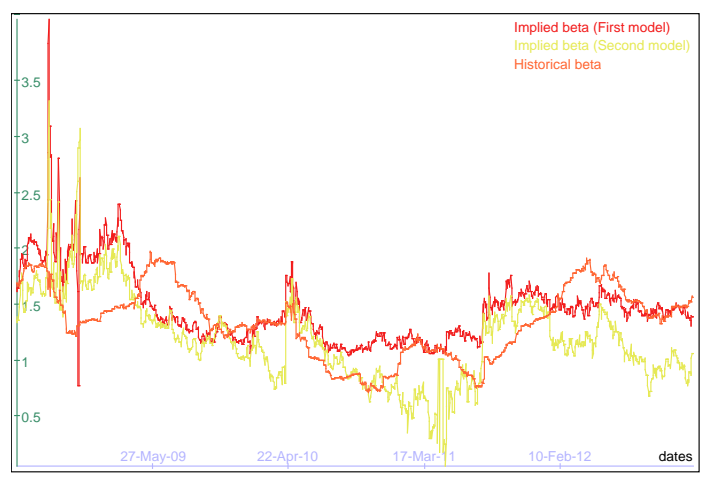

(f) GS vs SPX

Figure 2: Comparison between $\hat{\beta}, \tilde{\beta}$ and $\beta_{H}$ 


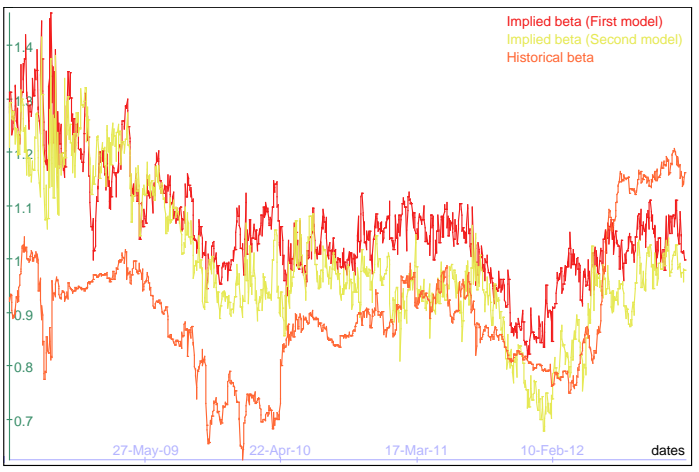

(a) MSFT vs SPX

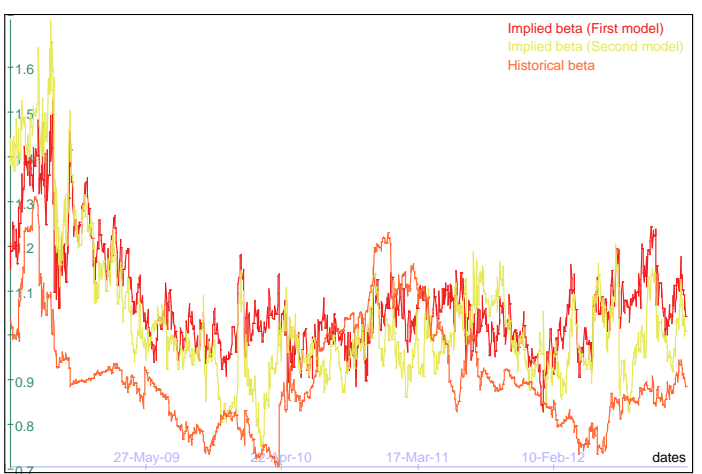

(c) GOOG vs SPX

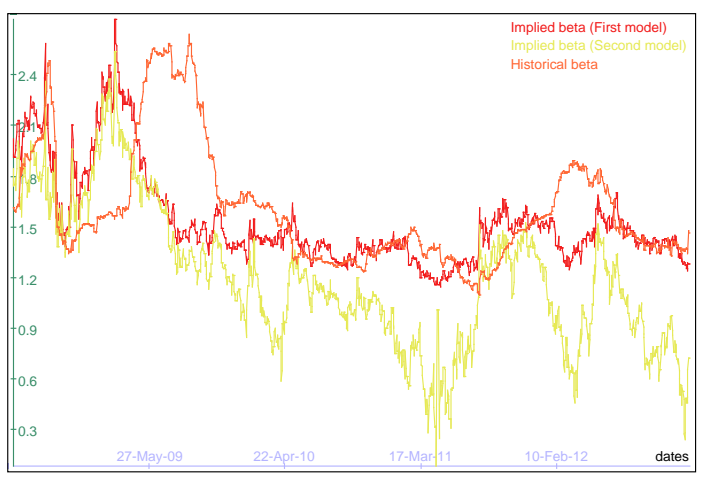

(e) JPM vs SPX

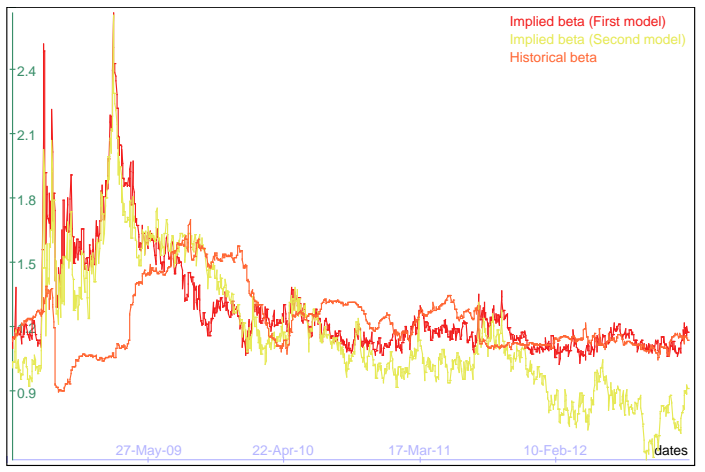

(b) GE vs SPX

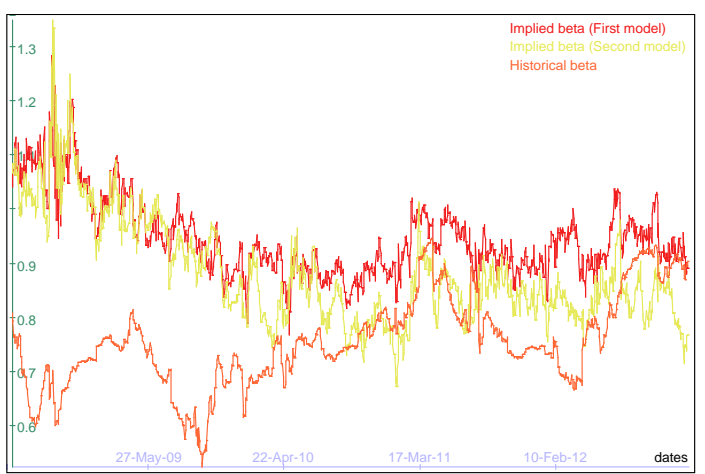

(d) IBM vs SPX

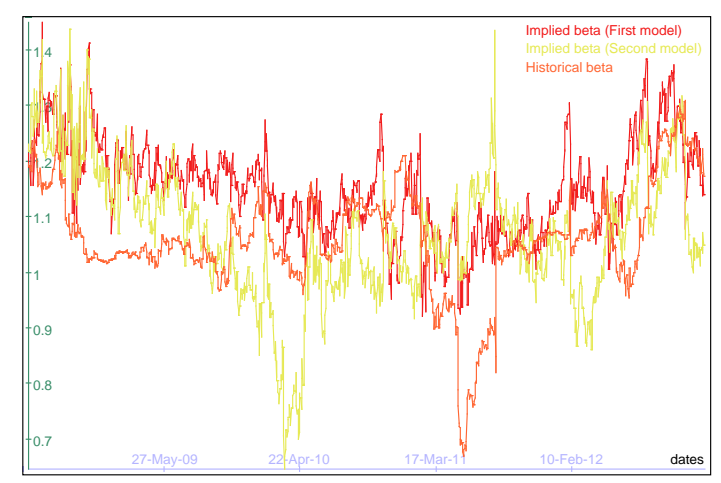

(f) CSCO vs SPX

Figure 3: Comparison between $\hat{\beta}, \tilde{\beta}$ and $\beta_{H}$ 


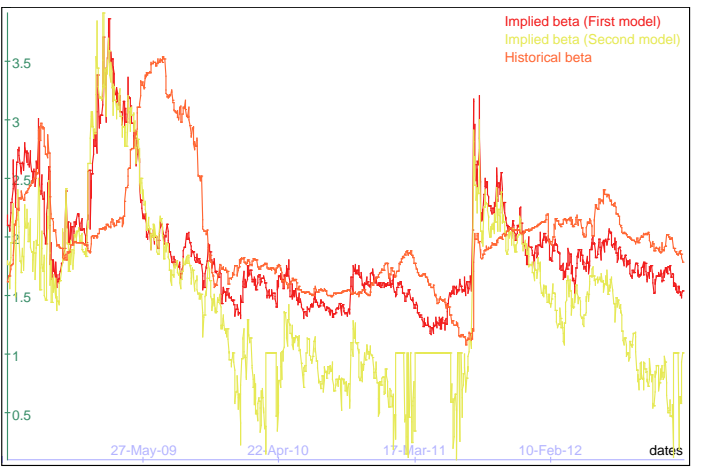

(a) BAC vs SPX

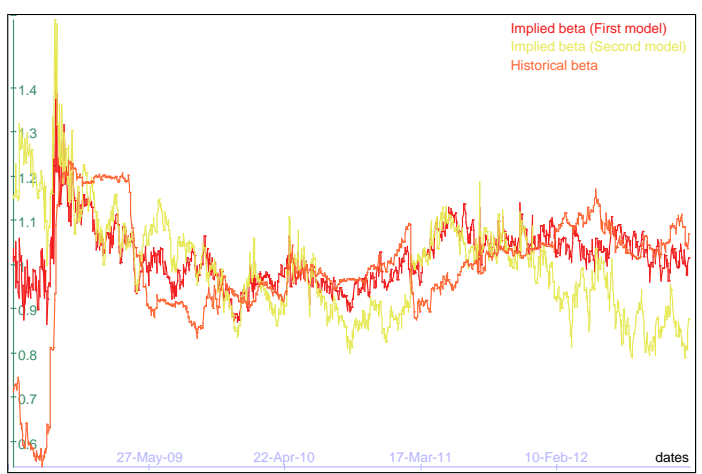

(c) CVX vs SPX

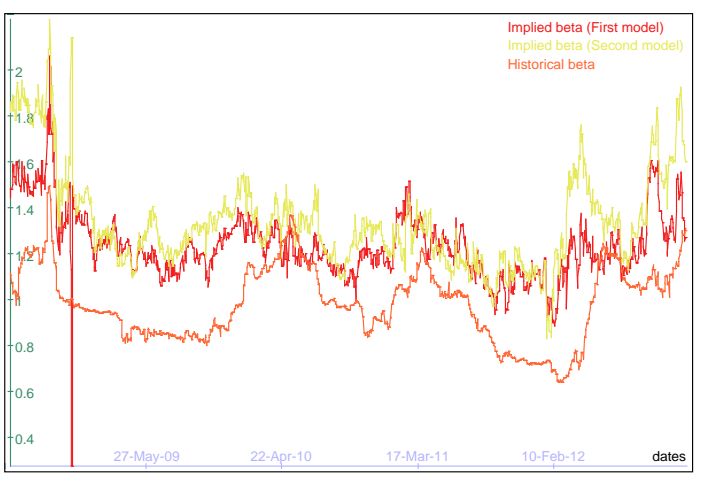

(e) AAPL vs SPX

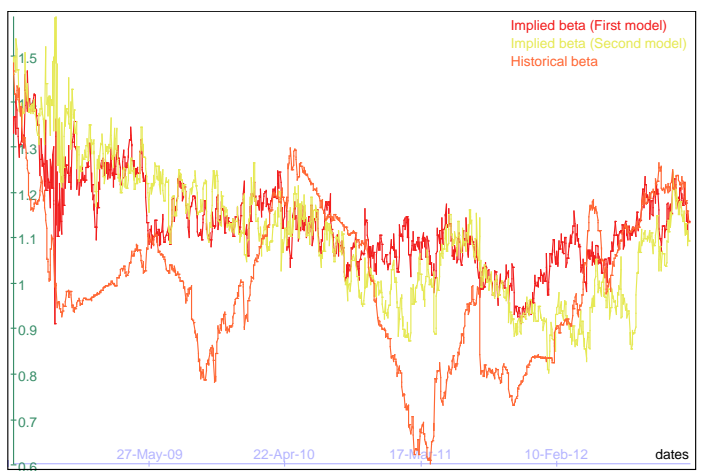

(b) INTC vs SPX

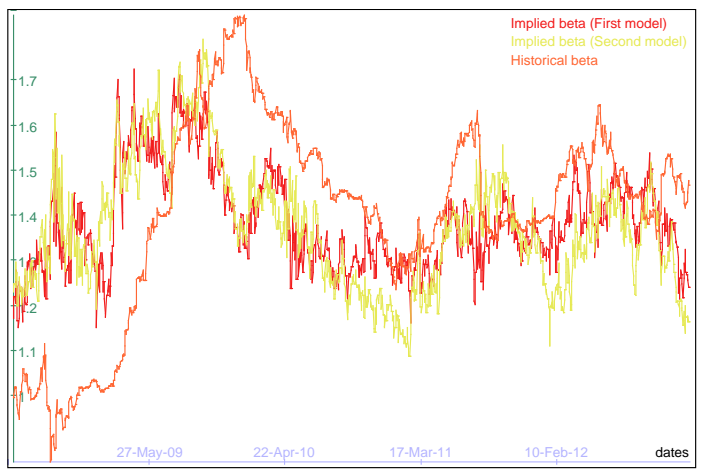

(d) CAT vs SPX

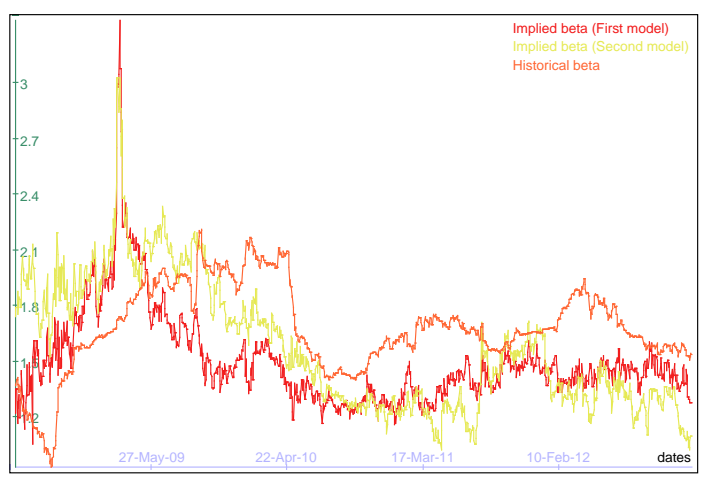

(f) AA vs SPX

Figure 4: Comparison between $\hat{\beta}, \tilde{\beta}$ and $\beta_{H}$ 
We define the parameter $\beta_{F}$ as the forward realized beta. At a given date $t, \beta_{F}(t)$ is defined as the slope of the linear regression of the stock log-returns on the index log-returns between $t$ and $t+T$. This means that $\beta_{F}(t)$ is a measure of the effective realization of the parameter $\beta$ on the forward window with length $T$.

We aim then to measure the prediction error relative to each of the estimators $\hat{\beta}, \tilde{\beta}$ and $\beta_{H}$. We use here the bias and the root-mean-square error as measures of the estimation error.

\begin{tabular}{cccc}
\hline \hline Stock & $E\left(\hat{\beta}-\beta_{F}\right)$ & $E\left(\tilde{\beta}-\beta_{F}\right)$ & $E\left(\beta_{H}-\beta_{F}\right)$ \\
\hline XLF.US & -0.148 & -0.121 & 0.054 \\
XLE.US & -0.05 & 0.0094 & -0.0313 \\
XLB.US & -0.0231 & 0.0002 & -0.0345 \\
XLK.US & 0.0679 & 0.0542 & -0.0158 \\
XLI.US & -0.0258 & -0.0183 & -0.0215 \\
AAPL.US & 0.2649 & 0.4073 & 0.0005 \\
AA.US & -0.1814 & -0.0738 & -0.0333 \\
BAC.US & -0.1414 & -0.3557 & 0.0222 \\
CAT.US & -0.0453 & -0.0574 & -0.0512 \\
CSCO.US & 0.0854 & 0.0216 & -0.0073 \\
CVX.US & 0.0252 & 0.0366 & -0.0187 \\
GE.US & 0.0375 & -0.0534 & 0.0016 \\
GOOG.US & 0.1693 & 0.1742 & 0.0193 \\
GS.US & 0.1306 & -0.0962 & 0.0113 \\
IBM.US & 0.2063 & 0.1575 & -0.0233 \\
INTC.US & 0.1462 & 0.1451 & -0.0069 \\
JPM.US & -0.0415 & -0.347 & 0.0383 \\
MSFT.US & 0.1717 & 0.1102 & -0.0234
\end{tabular}

Table 1: Bias of the estimators $\hat{\beta}, \tilde{\beta}$ and $\beta_{H}$ 


\begin{tabular}{cccc}
\hline \hline Stock & $\sqrt{E\left(\left(\frac{\hat{\beta}-\beta_{F}}{\beta_{F}}\right)^{2}\right)}$ & $\sqrt{E\left(\left(\frac{\tilde{\beta}-\beta_{F}}{\beta_{F}}\right)^{2}\right)}$ & $\sqrt{E\left(\left(\frac{\beta_{H}-\beta_{F}}{\beta_{F}}\right)^{2}\right)}$ \\
\hline XLF.US & 0.143 & 0.138 & 0.195 \\
XLE.US & 0.183 & 0.207 & 0.128 \\
XLB.US & 0.196 & 0.209 & 0.116 \\
XLK.US & 0.132 & 0.124 & 0.095 \\
XLI.US & 0.128 & 0.112 & 0.108 \\
AAPL.US & 0.41 & 0.547 & 0.252 \\
AA.US & 0.187 & 0.188 & 0.181 \\
BAC.US & 0.234 & 0.407 & 0.337 \\
CAT.US & 0.118 & 0.123 & 0.149 \\
CSCO.US & 0.159 & 0.162 & 0.147 \\
CVX.US & 0.198 & 0.238 & 0.165 \\
GE.US & 0.154 & 0.189 & 0.158 \\
GOOG.US & 0.277 & 0.31 & 0.165 \\
GS.US & 0.341 & 0.292 & 0.303 \\
IBM.US & 0.369 & 0.348 & 0.134 \\
INTC.US & 0.279 & 0.294 & 0.244 \\
JPM.US & 0.158 & 0.292 & 0.265 \\
MSFT.US & 0.297 & 0.27 & 0.163 \\
\hline
\end{tabular}

Table 2: RMSE of the estimators $\hat{\beta}, \tilde{\beta}$ and $\beta_{H}$

\subsection{Conclusion}

We can see through the tables above that the historical beta estimator $\beta_{H}$ is not biased for all the tested stocks and has in average the lowest RMSE compared to the two other estimators $\hat{\beta}$ and $\tilde{\beta}$. On the other hand, the forward looking beta estimator $\tilde{\beta}$ has a positive bias for the stocks AAPL, GOOG, IBM, INTC, MSFT and a negative bias for XLF and BAC. For those same stocks, the RMSE of the estimator $\tilde{\beta}$ is consequently higher than the one of $\beta_{H}$. This means that for some stocks, the market is expecting a realization of the parameter $\beta$ which is consistenty shifted to the effective realized one. We can say that for those stocks, the parameter $\beta$ is misspecified under the risk-neutral distribution. 


\section{Appendix}

\subsection{Appendix 1: Pricing options on the index}

Let $P_{t}^{I, \epsilon}=E\left(h\left(I_{T}\right) \mid I_{t}=x, Y_{t}=y\right)$ be the price of a european call on the index with strike $K$ and maturity $T$.

$$
P_{t}^{I, \epsilon}=P^{I, \epsilon}\left(t, I_{t}, Y_{t}\right)
$$

By applying Itô's lemma, we obtain:

$$
\mathcal{L}^{I} P_{t}^{I, \epsilon}=0 .
$$

We can then classify the elements of the generator $\mathcal{L}^{I}$ by powers of $\sqrt{\epsilon}$.

$$
\begin{aligned}
\mathcal{L}^{I} & =\mathcal{L}_{2}^{I}+\frac{1}{\sqrt{\epsilon}} \mathcal{L}_{1}^{I}+\frac{1}{\epsilon} \mathcal{L}_{0}^{I}, \\
\mathcal{L}_{0}^{I} & =\frac{\partial}{\partial y}\left(m_{Y}-y\right)+\nu_{Y}^{2} \frac{\partial^{2}}{\partial y^{2}} \\
\mathcal{L}_{1}^{I} & =-\nu_{Y} \sqrt{2} \chi_{1}(y) \frac{\partial}{\partial y}+\sqrt{2} \rho_{Y} \nu_{Y} I_{t} f_{1}(y) \frac{\partial^{2}}{\partial I \partial y}, \\
\mathcal{L}_{2}^{I} & =\frac{\partial}{\partial t}+r\left(\frac{\partial}{\partial I_{t}} I_{t}-.\right)+\frac{1}{2} \frac{\partial^{2}}{\partial I_{t}^{2}} I_{t}^{2} f_{1}(y)^{2} .
\end{aligned}
$$

We can notice here that $\mathcal{L}_{0}^{I}$ is the infinitesimal generator of the Ornstein-Uhlenbeck process $\left(Y_{1, t}\right)_{t}$ that diffuses according to the following SDE:

$$
d Y_{1, t}=\left(m_{Y}-Y_{1, t}\right) d t+\nu_{Y} \sqrt{2} d W_{t} .
$$

We expand then $P^{I, \epsilon}$ in powers of $\sqrt{\epsilon}$ :

$$
P^{I, \epsilon}=\sum_{i=0}^{\infty}(\sqrt{\epsilon})^{i} P_{i}^{I, \epsilon} .
$$

We can now do the expansion of the term $\mathcal{L}^{I} P_{t}^{I, \epsilon}=0$ and classify it by powers of $\sqrt{\epsilon}$. We give below the terms of orders $0,-1,-2,1$ respectively in $\sqrt{\epsilon}$.

$$
\begin{aligned}
& \text { (0) : } \mathcal{L}_{2}^{I} P_{0}^{I, \epsilon}+\mathcal{L}_{1}^{I} P_{1}^{I, \epsilon}+\mathcal{L}_{0}^{I} P_{2}^{I, \epsilon}=0, \\
& (-1): \mathcal{L}_{1}^{I} P_{0}^{I, \epsilon}+\mathcal{L}_{0}^{I} P_{1}^{I, \epsilon}=0, \\
& (-2): \mathcal{L}_{0}^{I} P_{0}^{I, \epsilon}=0 \text {, } \\
& \text { (1) : } \mathcal{L}_{2}^{I} P_{1}^{I, \epsilon}+\mathcal{L}_{1}^{I} P_{2}^{I, \epsilon}+\mathcal{L}_{0}^{I} P_{3}^{I, \epsilon}=0,
\end{aligned}
$$

We have $P_{0}^{I, \epsilon}$ is a solution of $\mathcal{L}_{0}^{I} P_{0}^{I, \epsilon}=0$ with final condition $P_{0}^{I, \epsilon}\left(T, I_{T}, y_{T}\right)=h\left(I_{T}\right)$ (independent of $\left.y_{T}\right)$. Then, by solving this equation, we find:

$$
P_{0}\left(t, I_{t}, y_{t}\right)=C_{1}\left(t, I_{t}\right) \int_{0}^{y} e^{\frac{u^{2}}{2 \mu_{Y}^{2}}-\frac{u m_{Y}}{\mu^{2}}} d u+C_{2}\left(t, I_{t}\right)
$$


If $C_{1}$ is not the null function then the solution diverges when $y \rightarrow+\infty$. We know that, in the case of a call option, we have $0 \leq P\left(t, I_{t}\right) \leq I_{t}$. Then we will choose $C_{1}$ to be null, and then $P_{0}$ is independent of $y$.

We have then $\mathcal{L}_{1}^{I} P_{0}^{I, \epsilon}+\mathcal{L}_{0}^{I} P_{1}^{I, \epsilon}=0$ which reduces to $\mathcal{L}_{0}^{I} P_{1}^{I, \epsilon}=0$ (since $P_{0}$ doesn't depend on $y)$. Using the same reasoning as before, we can prove that $P_{1}^{I, \epsilon}=P_{1}^{I, \epsilon}\left(t, I_{t}\right)$ is independent of $y$. Consequently, we have:

$$
\begin{aligned}
\mathcal{L}_{0}^{I} P_{0}^{I, \epsilon} & =\mathcal{L}_{1}^{I} P_{0}^{I, \epsilon}=0, \\
\mathcal{L}_{0}^{I} P_{1}^{I, \epsilon} & =\mathcal{L}_{1}^{I} P_{1}^{I, \epsilon}=0 .
\end{aligned}
$$

Consequently, the terms of order $(-1)$ and $(-2)$ in $\sqrt{\epsilon}$ are null. We focus now on the term of order 0 in $\sqrt{\epsilon}$. We have $: \mathcal{L}_{1}^{I} P_{1}^{I, \epsilon}=0$, then this term becomes:

$$
\mathcal{L}_{0}^{I} P_{2}^{I, \epsilon}+\mathcal{L}_{2}^{I} P_{0}^{I, \epsilon}=0 .
$$

This equation is a Poisson equation for $P_{2}^{I, \epsilon}$. The solvability condition for this equation is :

$$
<\mathcal{L}_{0}^{I} P_{2}^{I, \epsilon}>_{1}+<\mathcal{L}_{2}^{I} P_{0}^{I, \epsilon}>_{1}=0 .
$$

$<.>_{1}$ is then the average with respect to the invariant distribution $N\left(m_{Y}, \nu_{Y}^{2}\right)$ of the Ornstein-Uhlenbeck process $\left(Y_{1, t}\right)_{t}$.

Since $\mathcal{L}_{0}^{I}$ is the infinitesimal generator of the process $\left(Y_{1}\right)$, we can check easily that:

$$
<\mathcal{L}_{0}^{I} P_{2}^{I, \epsilon}>_{1}=0 .
$$

Then, the solvability condition reduces to:

$$
<\mathcal{L}_{2}^{I}>_{1} P_{0}^{I, \epsilon}=0
$$

We can then compute the average of the operator $\mathcal{L}_{2}^{I}$ :

$$
<\mathcal{L}_{2}^{I}>_{1}=\frac{\partial}{\partial t}+r\left(\frac{\partial}{\partial I} I-.\right)+\frac{1}{2} \frac{\partial^{2}}{\partial I^{2}} I^{2}<f_{1}^{2}>_{1} .
$$

If we note $\bar{\sigma}_{I}^{2}=<f_{1}^{2}>_{1}$ then $<\mathcal{L}_{2}^{I}>_{1}=\mathcal{L}_{B S}\left(\bar{\sigma}_{I}\right)$. We can then define $P_{0}^{I, \epsilon}$ as the solution to the following problem :

$$
\begin{aligned}
\mathcal{L}_{B S}\left(\bar{\sigma}_{I}\right) P_{0}^{I, \epsilon} & =0, \\
P_{0}^{\epsilon}\left(T, I_{T}\right) & =h\left(I_{T}\right) .
\end{aligned}
$$

We deduce then that $P_{0}^{I, \epsilon}=P_{B S}\left(t, I_{t}, \bar{\sigma}_{I}\right)$ meaning that $P_{0}^{I, \epsilon}$ is the Black-Scholes price of the index option with implied volatility equal to $\bar{\sigma}_{I}$.

We can then write $P_{2}^{I, \epsilon}$ as:

$$
P_{2}^{I, \epsilon}=-\left(\mathcal{L}_{0}^{I}\right)^{-1}\left(\mathcal{L}_{2}^{I}-<\mathcal{L}_{2}^{I}>_{1}\right) P_{0}^{I, \epsilon} .
$$

The term of order 1 in $\sqrt{\epsilon}$ is a poisson equation for $P_{3}^{I, \epsilon}$. The solvability condition for this equation is :

$$
\begin{aligned}
<\mathcal{L}_{2}^{I} P_{1}^{I, \epsilon}>_{1} & =-<\mathcal{L}_{1}^{I} P_{2}^{I, \epsilon}>_{1} \\
<\mathcal{L}_{2}^{I}>_{1} P_{1}^{I, \epsilon} & =<\mathcal{L}_{1}^{I}\left(\mathcal{L}_{0}^{I}\right)^{-1}\left(\mathcal{L}_{2}^{I}-<\mathcal{L}_{2}^{I}>_{1}\right)>P_{0}^{I, \epsilon}
\end{aligned}
$$


In order to solve the previous equation, we compute the difference between the operator $\mathcal{L}_{2}^{I}$ and its average:

$$
\mathcal{L}_{2}^{I}-<\mathcal{L}_{2}^{I}>_{1}=\frac{1}{2}\left(f_{1}^{2}(y)-<f_{1}^{2}>_{1}\right) I_{t}^{2} \frac{\partial^{2}}{\partial I_{t}^{2}} .
$$

Let $\phi_{I}$ the solution of:

$$
\mathcal{L}_{0} \phi_{I}(y)=f_{1}^{2}(y)-<f_{1}^{2}>_{1} .
$$

Using (5.4), we can write that:

$$
\left(\mathcal{L}_{0}^{I}\right)^{-1}\left(\mathcal{L}_{2}-<\mathcal{L}_{2}>_{1}\right)=\frac{1}{2} \phi_{I}(y) I_{t}^{2} \frac{\partial^{2}}{\partial I_{t}^{2}}
$$

By applying the operator $\mathcal{L}_{1}$ to the last equation, we get:

$$
\mathcal{L}_{1}^{I}\left(\mathcal{L}_{0}^{I}\right)^{-1}\left(\mathcal{L}_{2}-<\mathcal{L}_{2}>_{1}\right)=\left(-\nu_{Y} \sqrt{2}<\chi_{1} \phi_{I}^{\prime}>_{1}+\sqrt{2} \rho_{Y} \nu_{Y}<f_{1} \phi_{I}^{\prime}>_{1} I_{t} \frac{\partial}{\partial I_{t}}\right) \frac{1}{2} I_{t}^{2} \frac{\partial^{2}}{\partial I_{t}^{2}} .
$$

In order to simplify the notation, we define $V_{2}^{I, \epsilon}$ and $V_{3}^{I, \epsilon}$ as below:

$$
\begin{aligned}
V_{2}^{I, \epsilon} & =-\frac{\sqrt{\epsilon}}{\sqrt{2}} \nu_{Y}<\phi_{I}^{\prime} \chi_{1}>_{1}, \\
V_{3}^{I, \epsilon} & =\frac{\sqrt{\epsilon}}{\sqrt{2}} \rho_{Y} \nu_{Y}<\phi_{I}^{\prime} f>_{1} .
\end{aligned}
$$

Using $V_{2}^{I, \epsilon}$ and $V_{3}^{I, \epsilon}$, the equation (5.3) becomes:

$$
<\mathcal{L}_{2}^{I}>_{1} \sqrt{\epsilon} P_{1}^{I, \epsilon}=V_{2}^{I, \epsilon} I_{t}^{2} \frac{\partial^{2} P_{0}}{\partial I_{t}^{2}}+V_{3}^{I, \epsilon} I_{t} \frac{\partial}{\partial I_{t}}\left(I_{t}^{2} \frac{\partial^{2} P_{0}}{\partial I_{t}^{2}}\right)
$$

We can then deduce that $P_{1}^{I, \epsilon}$ is the solution for the following problem :

$$
\begin{aligned}
<\mathcal{L}_{2}^{I}>_{1} \sqrt{\epsilon} P_{1}^{I, \epsilon} & =V_{2}^{I, \epsilon} I_{t}^{2} \frac{\partial^{2} P_{0}}{\partial I_{t}^{2}}+V_{3}^{I, \epsilon} I_{t} \frac{\partial}{\partial I_{t}}\left(I_{t}^{2} \frac{\partial^{2} P_{0}}{\partial I_{t}^{2}}\right) \\
P_{1}^{I, \epsilon}\left(T, I_{T}\right) & =0 .
\end{aligned}
$$

We define the following operators:

$$
\begin{aligned}
\mathcal{D}_{1, I} & =I_{t} \frac{\partial}{\partial I_{t}} \\
\mathcal{D}_{2, I} & =I_{t}^{2} \frac{\partial^{2}}{\partial I_{t}^{2}} .
\end{aligned}
$$

Given that $<\mathcal{L}_{2}^{I}>_{1}=\mathcal{L}_{B S}\left(\bar{\sigma}_{I}\right)$ commits with $\mathcal{D}_{1, I}$ and $\mathcal{D}_{2, I}$, and that $\left\langle\mathcal{L}_{2}^{I}>_{1} P_{0}=0\right.$, the solution to the last problem can be given explicitly by:

$$
\sqrt{\epsilon} P_{1}^{I, \epsilon}=-(T-t)\left(V_{2}^{I, \epsilon} I_{t}^{2} \frac{\partial^{2} P_{0}}{\partial I_{t}^{2}}+V_{3}^{I, \epsilon} I_{t} \frac{\partial}{\partial I_{t}}\left(I_{t}^{2} \frac{\partial^{2} P_{0}}{\partial I_{t}^{2}}\right)\right) .
$$


If we neglect terms of order higher or equal to 2 in $\sqrt{\epsilon}$, we can approximate the option's price by $\left(P_{0}^{I, \epsilon}+\sqrt{\epsilon} P_{1}^{I, \epsilon}\right)$. As it was proven by Fouque et $\mathrm{Al}$ in $[6]$, we can carry out a parameter reduction method and approximate $P^{I, \epsilon}$ by the following formula:

$$
P^{I, \epsilon} \sim \tilde{P}_{0}^{I, \epsilon}+\sqrt{\epsilon} \tilde{P}_{1}^{I, \epsilon}
$$

such as:

$$
\begin{aligned}
\tilde{P}_{0}^{I, \epsilon} & =P_{B S}\left(\bar{\sigma}_{I}^{*}\right) \\
\left(\bar{\sigma}_{I}^{*}\right)^{2} & =\bar{\sigma}_{I}^{2}-2 V_{2}^{I, \epsilon}, \\
\sqrt{\epsilon} \tilde{P}_{1}^{I, \epsilon} & =-(T-t) V_{3}^{I, \epsilon} I_{t} \frac{\partial}{\partial I_{t}}\left(I_{t}^{2} \frac{\partial^{2} \tilde{P}_{0}}{\partial I_{t}^{2}}\right) .
\end{aligned}
$$

\subsection{Appendix 2: Accuracy of the approximation}

We want to prove here that the approximation $\left(P_{0}^{S, \epsilon}+\sqrt{\epsilon} P_{1}^{S, \epsilon}\right)$ of the price $P\left(t, S_{t}, Y_{t}\right)$ is of order 1 in $\epsilon$ meaning that:

$$
\left|P\left(t, S_{t}, Y_{t}\right)-\left(P_{0}^{S, \epsilon}+\sqrt{\epsilon} P_{1}^{S, \epsilon}\right)\right| \leq C \epsilon .
$$

The proof of this property is given in [10] in the case where the payoff $h$ is smooth. We will give here a summary of this proof in order to make this paper self contained.

Let us introduce the quantity $Z^{S, \epsilon}$ which verifies that:

$$
P\left(t, S_{t}, Y_{t}\right)=P_{0}^{S, \epsilon}+\sqrt{\epsilon} P_{1}^{S, \epsilon}+\epsilon P_{2}^{S, \epsilon}+\epsilon^{\frac{3}{2}} P_{3}^{S, \epsilon}-Z^{S, \epsilon} .
$$

Given that $\mathcal{L}^{S} P\left(t, S_{t}, Y_{t}\right)=0$, we have:

$$
\mathcal{L}^{S} Z^{S, \epsilon}=\mathcal{L}_{S}\left(P_{0}^{S, \epsilon}+\sqrt{\epsilon} P_{1}^{S, \epsilon}+\epsilon P_{2}^{S, \epsilon}+\epsilon^{\frac{3}{2}} P_{3}^{S, \epsilon}\right)
$$

We recall that $\mathcal{L}^{S}=\mathcal{L}_{2}^{S}+\frac{1}{\sqrt{\epsilon}} \mathcal{L}_{1}^{S}+\frac{1}{\epsilon} \mathcal{L}_{0}^{S}$. We can develop then $\mathcal{L}^{S} Z^{S, \epsilon}$ and regroup the terms by orders of $\sqrt{\epsilon}$ :

$$
\begin{aligned}
\mathcal{L}^{S} Z^{S, \epsilon} & =\frac{1}{\epsilon} \mathcal{L}_{0}^{S} P_{0}^{S, \epsilon}+\frac{1}{\sqrt{\epsilon}}\left(\mathcal{L}_{0}^{S} P_{1}^{S, \epsilon}+\mathcal{L}_{1}^{S} P_{0}^{S, \epsilon}\right)+\left(\mathcal{L}_{0}^{S} P_{2}^{S, \epsilon}+\mathcal{L}_{1}^{S} P_{1}^{S, \epsilon}+\mathcal{L}_{2}^{S} P_{0}^{S, \epsilon}\right) \\
& +\sqrt{\epsilon}\left(\mathcal{L}_{0}^{S} P_{3}^{S, \epsilon}+\mathcal{L}_{1}^{S} P_{2}^{S, \epsilon}+\mathcal{L}_{2}^{S} P_{1}^{S, \epsilon}\right)+\epsilon\left(\mathcal{L}_{1}^{S} P_{3}^{S, \epsilon}+\mathcal{L}_{2}^{S} P_{2}^{S, \epsilon}\right)+\epsilon^{\frac{3}{2}} \mathcal{L}_{2}^{S} P_{3}^{S, \epsilon} .
\end{aligned}
$$

Since $P_{0}^{S, \epsilon}, P_{1}^{S, \epsilon}$ and $P_{2}^{S, \epsilon}$ are chosen to nullify the first four terms in the previous equation. Then we obtain:

$$
\mathcal{L}^{S} Z^{S, \epsilon}=\epsilon\left(\mathcal{L}_{1}^{S} P_{3}^{S, \epsilon}+\mathcal{L}_{2}^{S} P_{2}^{S, \epsilon}\right)+\epsilon^{\frac{3}{2}} \mathcal{L}_{2}^{S} P_{3}^{S, \epsilon},
$$

with the final condition:

$$
Z^{S, \epsilon}\left(T, S_{T}, Y_{T}, Z_{T}\right)=\epsilon P_{2}\left(T, S_{T}, Y_{T}, Z_{T}\right)+\epsilon^{\frac{3}{2}} P_{3}\left(T, S_{T}, Y_{T}, Z_{T}\right)
$$


Using the Feynman-Kac theorem, we obtain:

$$
\begin{aligned}
Z^{S, \epsilon}(t, x, y, z) & =\epsilon E\left(e^{-r(T-t)}\left(P_{2}\left(T, S_{T}, Y_{T}, Z_{T}\right)+\epsilon^{\frac{1}{2}} P_{3}\left(T, S_{T}, Y_{T}, Z_{T}\right)\right)\right. \\
& \left.-\int_{t}^{T} e^{-r(u-t)}\left(\left(\mathcal{L}_{1}^{S} P_{3}^{S, \epsilon}+\mathcal{L}_{2}^{S} P_{2}^{S, \epsilon}\right)+\epsilon^{\frac{1}{2}} \mathcal{L}_{2}^{S} P_{3}^{S, \epsilon}\right)\left(u, S_{u}, Y_{u}, Z_{u}\right) d u \mid S_{t}=x, Y_{t}=y, Z_{t}=z\right)
\end{aligned}
$$

Under the following assumptions:

- The payoff function $h$ is smooth.

- The functions $f, \chi_{1}$ and $\chi_{2}$ are bounded, so that the solutions of the poisson equation are at most linearly growing in $|y|$ and $|z|$

We can deduce that $Z^{S, \epsilon}$ is at most linearly growing in $|y|$ and $|z|$ and bounded uniformly in $x$, and we have:

$$
Z^{S, \epsilon}(t, x, y, z)=O(\epsilon) .
$$

Consequently: $P\left(t, S_{t}, Y_{t}, Z_{t}\right)=P_{0}^{S, \epsilon}+\sqrt{\epsilon} P_{1}^{S, \epsilon}+O(\epsilon)$. In order to drop the condition on the boundedness of the payoff function $h$, we should have $\epsilon$ independent estimates for moments of $S^{\epsilon}$. This would be the case when the processes $Y$ and $Z$ have enougn time to fluctuate in order to attain respectively their invariant distributions. Consequently, when the payoff function is not bounded, the approximation $P_{0}^{S, \epsilon}+\sqrt{\epsilon} P_{1}^{S, \epsilon}$ is of order 1 in $\epsilon$ when the maturity of the option (of the payoff function $h$ ) is sufficiently larger than $\epsilon$.

The demonstration of the accuracy of the approximation for a non smooth payoff $h$ (as in the case of a call option) is derived in [14.

\section{References}

[1] Marshall E Blume and Irwin Friend. A new look at the capital asset pricing model. The journal of finance, 28(1):19-34, 1973.

[2] John Y Campbell, Martin Lettau, Burton G Malkiel, and Yexiao Xu. Have individual stocks become more volatile? an empirical exploration of idiosyncratic risk. The Journal of Finance, 56(1):1-43, 2001.

[3] Peter Carr and Dilip Madan. Factor models for option pricing. forthcoming, Asia Pacific Financial Markets, 2000.

[4] Peter Christoffersen, Kris Jacobs, and Gregory Vainberg. Forward-looking betas. manuscript, McGill University, 2008.

[5] Eugene F Fama and Kenneth R French. The capital asset pricing model: theory and evidence. Journal of Economic Perspectives, pages 25-46, 2004.

[6] Jean-Pierre Fouque and Eli Kollman. Calibration of stock betas from skews of implied volatilities. Applied Mathematical Finance, 18(2):119-137, 2011. 
[7] Jean-Pierre Fouque and Adam P Tashman. Option pricing under a stressed-beta model. Annals of Finance, 8(2-3):183-203, 2012.

[8] José Carlos Garcia Franco. Maximum likelihood estimation of mean reverting processes. Real Options Practice, 2003.

[9] Don UA Galagedera. An alternative perspective on the relationship between downside beta and capm beta. Emerging Markets Review, 8(1):4-19, 2007.

[10] Foque Jean-Peirre, George Papanicolaou, and K Ronnie Sircar. Derivatives in financial markets with stochastic volatility. Cambridge University Press, 2000.

[11] Colm Kearney and Valerio Potì. Have european stocks become more volatile? an empirical investigation of idiosyncratic and market risk in the euro area. European Financial Management, 14(3):419-444, 2008.

[12] Harry Markowitz. Portfolio selection*. The journal of finance, 7(1):77-91, 1952.

[13] Asset Pricing Model. Investment analysis \& portfolio management. 1967.

[14] George Papanicolaou, Jean-Pierre Fouque, Knut Solna, and Ronnie Sircar. Singular perturbations in option pricing. SIAM Journal on Applied Mathematics, 63(5):16481665, 2003.

[15] Steven X Wei and Chu Zhang. Why did individual stocks become more volatile?*. The Journal of Business, 79(1):259-292, 2006.

[16] J Fred Weston. Investment decisions using the capital asset pricing model. Financial Management, pages 25-33, 1973.

[17] Yexiao Xu and Burton G Malkiel. Investigating the behavior of idiosyncratic volatility*. The Journal of Business, 76(4):613-645, 2003.

[18] Yan Zhang. Estimation of beta in a simple functional capital asset pricing model for high frequency us stock data. 2011. 